\title{
Algorithms for Omega-Regular Games with Incomplete Information
}

\author{
Krishnendu Chatterjee \\ Laurent Doyen \\ Thomas A. Henzinger \\ Jean-Francois Raskin
}

Electrical Engineering and Computer Sciences University of California at Berkeley

Technical Report No. UCB/EECS-2006-89

http://www.eecs.berkeley.edu/Pubs/TechRpts/2006/EECS-2006-89.html

June 26, 2006 
Copyright @ 2006, by the author(s).

All rights reserved.

Permission to make digital or hard copies of all or part of this work for personal or classroom use is granted without fee provided that copies are not made or distributed for profit or commercial advantage and that copies bear this notice and the full citation on the first page. To copy otherwise, to republish, to post on servers or to redistribute to lists, requires prior specific permission.

\section{Acknowledgement}

This research was supported in part by the NSF grants CCR-0225610 and CCR-0234690, and by the SNSF under the Indo-Swiss Joint Research Programme. 


\title{
Algorithms for Omega-Regular Games with Incomplete Information *
}

\author{
Krishnendu Chatterjee ${ }^{1} \quad$ Laurent Doyen $^{2}$ \\ Thomas A. Henzinger ${ }^{1,3}$ \\ Jean-François Raskin ${ }^{2}$ \\ ${ }^{1}$ EECS, University of California at Berkeley, U.S.A. \\ ${ }^{2}$ CS, Université Libre de Bruxelles, Belgium \\ ${ }^{3}$ CCS, École Polytechnique Fédérale de Lausanne, Switzerland
}

June 26, 2006

\begin{abstract}
We study observation-based strategies for two-player turn-based games on graphs with omega-regular objectives. An observation-based strategy relies on incomplete information about the history of a play, namely, on the past sequence of observations. Such games occur in the synthesis of a controller that does not see the private state of the plant. Our main results are twofold. First, we give a fixpoint algorithm for computing the set of states from which a player can win with a deterministic observation-based strategy for any omega-regular objective. The fixpoint is computed in the lattice of antichains of state sets. This algorithm has the advantages of being directed by the objective and of avoiding an explicit subset construction on the game graph. Second, we give an algorithm for computing the set of states from which a player can win with probability 1 with a randomized observationbased strategy for a Büchi objective. This set is of interest because in the absence of complete information, randomized strategies are more powerful than deterministic ones. We show that our algorithms are optimal by proving matching lower bounds.
\end{abstract}

${ }^{*}$ This research was supported in part by the NSF grants CCR-0225610 and CCR0234690, and by the SNSF under the Indo-Swiss Joint Research Programme. 


\section{Introduction}

Two-player games on graphs play an important role in computer science. In particular, the controller synthesis problem asks, given a model for a plant, to construct a model for a controller such that the behaviors resulting from the parallel composition of the two models respects a given specification (e.g., are included in an $\omega$-regular set). Controllers can be synthesized as winning strategies in a graph game whose vertices represent the plant states, and whose players represent the plant and the controller [17, 18]. Other applications of graph games include realizability and compatibility checking, where the players represent parallel processes of a system, or its environment $[1,11,6]$.

Most results about two-player games played on graphs make the hypothesis of complete information. In this setting, the controller knows, during its interaction with the plant, the exact state of the plant. In practice, this hypothesis is often not reasonable. For example, in the context of hybrid systems, the controller acquires information about the state of the plant using sensors with finite precision, which return incomplete information about the state. Similarly, if the players represent individual processes, then a process has only access to the public variables of the other processes, not to their private variables $[19,2]$.

Two-player games of incomplete information are considerably more complicated than games of complete information. First, decision problems for incomplete-information games usually lie in higher complexity classes than their complete-information counter-parts $[19,14,2]$. The algorithmic difference is often exponential, due to a subset construction that, similar to the determinization of finite automata, turns an incomplete-information game into an equivalent complete-information game. Second, because of the determinization, no symbolic algorithms are known to solve incompleteinformation games. This is in contrast to the complete-information case, where (often) simple and elegant fixed-point algorithms exist [12, 8]. Third, in the context of incomplete information, deterministic strategies are sometimes insufficient. A game is turn-based if in every state one of the players chooses a successor state. While deterministic strategies suffice to win turnbased games of complete information, turn-based games of incomplete information require randomized strategies to win with probability 1 . Fourth, winning strategies for incomplete-information games need memory even for simple objectives such as safety and reachability. This is again in contrast to the complete-information case, where turn-based safety and reachability games can be won with memoryless strategies. We will illustrate the needs 
for randomization and memory with an example.

The contributions of this paper are two-fold. First, we provide a symbolic fixed-point algorithm to solve games of incomplete information for arbitrary $\omega$-regular objectives. The novelty is that our algorithm is symbolic; it does not carry out an explicit subset construction. Instead, we compute fixed points on the lattice of antichains of state sets. Antichains of state sets can be seen as a symbolic and compact representation for $\subseteq$-downward-closed sets of sets of states. ${ }^{1}$ This solution extends our recent result [10] from safety objectives to all $\omega$-regular objectives. To justify the correctness of the algorithm, we transform games of incomplete information into games of complete information while preserving the existence of winning strategies for every Borel objective. The reduction is only part of the proof, not part of the algorithm. For the special case of parity objectives, we obtain a symbolic EXPTIME algorithm for solving parity games of incomplete information. This is optimal as the reachability problem for games of incomplete information is known to be ExPTIme-hard [19].

Second, we study randomized strategies and winning with probability 1 for incomplete-information games. To our knowledge, for these games no algorithms (symbolic or not) are present in the literature. Following [7], we refer to winning with probability 1 as almost-sure winning (almost winning, for short), in contrast to sure winning with deterministic strategies. We provide a symbolic ExPTIME algorithm to compute the set of almostwinning states for games of incomplete information with Büchi objectives (reachability objectives can be obtained as a special case and for safety objectives almost-winning and sure-winning coincide). Our solution is again justified by a reduction to games of complete information. However, for randomized strategies the reduction is different, and considerably more complicated. We prove our algorithm to be optimal, showing that computing the almost-winning states for reachability games of incomplete information is EXPTIME-hard.

The structure of the paper is simple: Section 2 presents the definitions; Section 3 gives the algorithm for the case of sure winning with deterministic strategies; Section 4, for the case of almost winning with randomized strategies; and Section 5 provides the lower bounds.

\footnotetext{
${ }^{1}$ We have recently used this symbolic representation of $\subseteq$-downward-closed sets of state sets to propose a new algorithm to solve the universality problem for nondeterministic finite automata. First experiments show very promising performances, see [9] for details.
} 
Related work. In [17], Pnueli and Rosner study the synthesis of reactive modules. In their framework, there is no game graph; instead, the environment and the objective are specified using an LTL formula. In [14], Kupferman and Vardi extend these results in two directions: they consider $\mathrm{CTL}^{*}$ objectives and incomplete information. Again, no game graph, but a specification formula is given to the synthesis procedure. We believe that our setting, where a game graph is given explicitly, is more suited to fully and uniformly understand the role of incomplete information. Indeed, Kupferman and Vardi assert that incomplete information comes at no cost, because if the specification is given as a CTL (or CTL*) formula, then the synthesis problem is complete for ExPTIme (resp., 2ExPTIME), just as in the complete-information case. These hardness results, however, depend on the fact that the specification is given compactly as a formula. In our setting, with an explicit game graph, reachability games of complete information are known to be PTIME-complete, whereas we know ExPTIME-completeness for reachability games of incomplete information. In [19], Reif studies the complexity of sure winning for graph games of incomplete information with simple reachability objectives. The solution proposed by Reif comes in the form of an explicit subset construction. The hardness proof uses private and blind alternating turing machines. None of the above works provide symbolic solutions, and none of them consider randomized strategies.

It is known that for Partially Observable Markov Decision Processes (POMDPs) with boolean rewards and limit-average objectives the quantitative analysis (whether the value is greater than a specified value) is EXPTIME-complete, see [15]. However, the almost-winning is a qualitative question and the hardness result for almost-winning for incomplete information games does not follow from those results on POMDPs. We propose in section 5 an alternative proof of hardness for games of incomplete information with reachability objectives and we show that this result extends to almost winning conditions as well. To the best of our knowledge, this is the first hardness result that applies to qualitative analysis of almost-winning in incomplete information games.

A sub-class of semi-perfect (or semi-complete) information games (where only one player has incomplete information and the other player has complete information) is studied in [4]. The class of games in [4] is simpler than the class of games we study and can be solved in NP $\cap$ coNP for parity objectives. 


\section{Definitions}

Games. A game structure of incomplete information is a tuple $G=$ $\left\langle L, l_{0}, \Sigma, \Delta\right.$, Obs, $\left.\gamma\right\rangle$ where $L$ is a finite set of states, $l_{0} \in L$ is the initial state, $\Sigma$ is a finite alphabet, $\Delta \subseteq L \times \Sigma \times L$ is a set of labeled transitions, Obs is a finite set of observations, and $\gamma$ : Obs $\rightarrow 2^{L} \backslash \emptyset$ maps each observation to the set of states that it represents. Furthermore, we require the following properties on $G$ : $(i)$ for all $\ell \in L$, for all $\sigma \in \Sigma$, there exists $\ell^{\prime} \in L$ : $\left(\ell, \sigma, \ell^{\prime}\right) \in \Delta ;(i i)$ the set of sets $\{\gamma(o) \mid o \in$ Obs $\}$ partitions $L$. The size of $G$ is $|L|$. We say that $G=\left\langle L, l_{0}, \Sigma, \Delta\right.$, Obs, $\left.\gamma\right\rangle$ is a game structure of complete information if Obs $=L$ and $\gamma(\ell)=\{\ell\}$ for all $\ell \in L$. We often omit (Obs, $\gamma$ ) in the description of games of complete information. For $\sigma \in \Sigma$ and $s \subseteq L$ let $\operatorname{Post}_{\sigma}^{G}(s)=\left\{\ell^{\prime} \in L \mid \exists \ell \in s:\left(\ell, \sigma, \ell^{\prime}\right) \in \Delta\right\}$.

Plays and observations. In a game structure, in each turn, Player 1 chooses a letter in $\Sigma$ and Player 2 resolves nondeterminism by choosing the successor. A play in $G$ is an infinite sequence $\pi=\ell_{0} \sigma_{0} \ell_{1} \ldots \sigma_{n-1} \ell_{n} \sigma_{n} \ldots$ such that $(i) \ell_{0}=l_{0}$, and $(i i)$ for all $i \geq 0$ : $\left(\ell_{i}, \sigma_{i}, \ell_{i+1}\right) \in \Delta$. The set of plays in $G$ is noted Play $(G)$. Fix a play $\pi=\ell_{0} \sigma_{0} \ell_{1} \ldots \sigma_{n-1} \ell_{n} \sigma_{n} \ldots$ in $G$. The prefix up to $\ell_{n}$ of $\pi$ is denoted by $\pi(n)$, i.e., it is the finite sequence $\ell_{0} \sigma_{0} \ell_{1} \ldots \sigma_{n-1} \ell_{n}$. its length is $|\pi(n)|=n+1$ and its last element is $\operatorname{Last}(\pi(n))=\ell_{n}$. The observation of $\pi$ is the unique infinite sequence $\operatorname{Obs}(\pi)=o_{0} \sigma_{0} o_{1} \ldots \sigma_{n-1} o_{n} \sigma_{n} \ldots$ such that for all $i \geq 0$, $\ell_{i} \in \gamma\left(o_{i}\right)$. The observation of $\pi(n)$ is the prefix up to $o_{n}$ of $\operatorname{Obs}(\pi)$. We define similarly the observation of prefixes. The set of plays in $G$ is noted Play $(G)$ and the set of prefixes in $G$ is noted $\operatorname{Pref}(G)$. A state $\ell \in L$ is reachable in $G$ if there exists a prefix $\rho \in \operatorname{Pref}(G)$ such that $\operatorname{Last}(\rho)=\ell$. Let $\rho=\ell_{0} \sigma_{0} \ell_{1} \ldots \sigma_{n-1} \ell_{n} \in \operatorname{Pref}(G)$, the observation of $\rho$, noted $\operatorname{Obs}(\rho)$, is the unique sequence $o_{0} \sigma_{0} o_{1} \ldots \sigma_{n-1} o_{n}$ such that for all $i, 0 \leq i \leq n, \ell_{i} \in \gamma\left(o_{i}\right)$. Given $\rho \in \operatorname{Pref}(G)$, we define $\left[\rho \rrbracket_{\text {Obs }}\right.$ as the set of prefixes of plays in $G$ that have the same observation as $\rho$ : $[\rho]_{\text {Obs }}=\left\{\rho^{\prime} \mid \rho^{\prime} \in \operatorname{Pref}(G)\right.$ and $\left.\operatorname{Obs}(\rho)=\operatorname{Obs}\left(\rho^{\prime}\right)\right\}$. The knowledge associated to a sequence of observations $\tau=o_{0} \sigma_{0} o_{1} \sigma_{1} \ldots \sigma_{n-1} o_{n}$, noted $\mathrm{K}(\tau)$, is the set of states in which the play can be after this sequence of observations: $\mathrm{K}(\tau)=\{\operatorname{Last}(\rho) \mid \rho \in \operatorname{Pref}(G)$ and $\operatorname{Obs}(\rho)=\tau\}$.

Given $\rho \in \operatorname{Pref}(G)$, we define $[\rho]_{K}$ as the set of prefixes of plays in $G$ that leads to the same knowledge: $[\rho]_{K}=\left\{\rho_{1} \in \operatorname{Pref}(G) \mid \mathrm{K}\left(\operatorname{Obs}\left(\rho_{1}\right)\right)=\right.$ $\mathrm{K}(\mathrm{Obs}(\rho))\}$. 
Lemma 1 Let $G=\left\langle L, l_{0}, \Sigma, \Delta\right.$, Obs, $\left.\gamma\right\rangle$ be a game structure of incomplete information. For $\sigma \in \Sigma, \ell \in L$ and $\rho, \rho^{\prime} \in \operatorname{Pref}(G)$ with $\rho^{\prime}=\rho \cdot \sigma \cdot \ell$, let $\mathrm{obs}_{\ell} \in \mathrm{Obs}$ be the unique observation such that $\ell \in \gamma\left(\mathrm{obs}_{\ell}\right)$. Then we have $\mathrm{K}\left(\operatorname{Obs}\left(\rho^{\prime}\right)\right)=\operatorname{Post}_{\sigma}^{G}(\mathrm{~K}(\operatorname{Obs}(\rho))) \cap \gamma\left(\right.$ obs $\left._{\ell}\right)$.

Strategies. Let $G=\left\langle L, l_{0}, \Sigma, \Delta\right.$, Obs, $\left.\gamma\right\rangle$ be a game structure of incomplete information. A deterministic strategy in $G$ for Player 1 is a function $\alpha: \operatorname{Pref}(G) \rightarrow \Sigma$. For a finite set $A$, a probability distribution on $A$ is a function $\kappa: A \rightarrow[0,1]$ such that $\sum_{a \in A} \kappa(a)=1$. We denote the set of probability distributions on $A$ by $\mathcal{D}(A)$. Given a distribution $\kappa \in \mathcal{D}(A)$, let Supp $(\kappa)=\{a \in A \mid \kappa(a)>0\}$ the support of $\kappa$. A randomized strategy in $G$ for Player 1 is a function $\alpha: \operatorname{Pref}(G) \rightarrow \mathcal{D}(\Sigma)$. A (deterministic or randomized) strategy $\alpha$ for Player 1 is said to be observation-based if for all prefixes $\rho, \rho^{\prime} \in \operatorname{Pref}(G)$, if $\operatorname{Obs}(\rho)=\operatorname{Obs}\left(\rho^{\prime}\right)$ then $\alpha(\rho)=\alpha\left(\rho^{\prime}\right)$. In the sequel, we are interested in the existence of observation-based strategies for Player 1 . A deterministic strategy in $G$ for Player 2 is a function $\beta: \operatorname{Pref}(G) \times \Sigma \rightarrow L$ such that $\forall \rho \in \operatorname{Pref}(G) \cdot \forall \sigma \in \Sigma:(\operatorname{Last}(\rho), \sigma, \beta(\rho, \sigma)) \in \Delta$. A randomized strategy in $G$ for Player 2 is a function $\beta: \operatorname{Pref}(G) \times \Sigma \rightarrow \mathcal{D}(L)$ such that $\forall \rho \in \operatorname{Pref}(G) \cdot \forall \sigma \in \Sigma \cdot \forall \ell \in \operatorname{Supp}(\beta(\rho, \sigma)):(\operatorname{Last}(\rho), \sigma, \ell) \in \Delta$. We denote by $\mathcal{A}_{G}, \mathcal{A}_{G}^{O}$ and $\mathcal{B}_{G}$ the set of all Player 1 strategies, the set of all observationbased Player 1 strategies, and the set of all Player 2 strategies, respectively. All the results of the paper can also be proved if strategies are mappings from sequence of states only (without the moves).

The outcome of two deterministic strategies $\alpha$ (for Player 1 ) and $\beta$ (for Player 2) in $G$ is the play $\pi=\ell_{0} \sigma_{0} \ell_{1} \ldots \sigma_{n-1} \ell_{n} \sigma_{n} \ldots \in \operatorname{Play}(G)$ such that for all $i \geq 0$, we have $\sigma_{i}=\alpha(\pi(i))$ and $\ell_{i+1}=\beta\left(\pi(i), \sigma_{i}\right)$. This play is noted outcome $(G, \alpha, \beta)$. The outcome of two randomized strategies $\alpha$ (for Player 1) and $\beta$ (for Player 2) in $G$ is the set of plays $\pi=\ell_{0} \sigma_{0} \ell_{1} \ldots \sigma_{n-1} \ell_{n} \sigma_{n} \cdots \in \operatorname{Play}(G)$ such that for all $i \geq 0$, we have $\alpha(\pi(i))\left(\sigma_{i}\right)>0$ and $\beta\left(\pi(i), \sigma_{i}\right)\left(l_{i+1}\right)>0$. The outcome set of the deterministic (resp. randomized) strategy $\alpha$ for Player 1 in $G$ is the set Outcome ${ }_{1}(G, \alpha)$ of plays outcome $(G, \alpha, \beta)$ such that $\beta$ is a deterministic (resp. randomized) strategy for Player 2. Similarly, the outcome set of the deterministic (resp. randomized) strategy $\beta$ for Player 2 in $G$ is the set Outcome $_{2}(G, \beta)$ of plays outcome $(G, \alpha, \beta)$ such that $\alpha$ is a deterministic (resp. randomized) strategy for Player 1.

Objectives. An objective in $G$ is a set $\phi$ of infinite sequences of observations and input letters, i.e. $\phi \subseteq(\mathrm{Obs} \times \Sigma)^{\omega}$. A play $\pi=$ 
$\ell_{0} \sigma_{0} \ell_{1} \ldots \sigma_{n-1} \ell_{n} \sigma_{n} \ldots \in$ Play $(G)$ satisfies an objective $\phi$ (noted $\pi \models \phi$ ) iff $\operatorname{Obs}(\pi) \in \phi$. Objectives are generally Borel measurable; a Borel objective is a Borel set in the Cantor topology on $(\text { Obs } \times \Sigma)^{\omega}[13]$. We also consider reachability, safety, (open and closed sets in the Cantor topology on $(\mathrm{Obs} \times \Sigma)^{\omega}$, respectively) Büchi, coBüchi and parity objectives. The parity objectives are a canonical form to express all $\omega$-regular objectives [20]. For a play $\pi=$ $\ell_{0} \sigma_{0} \ell_{1} \ldots$ we denote by $\operatorname{Inf}(\pi)$ the set of observations that appear infinitely often in $\operatorname{Obs}(\pi)$, i.e., $\operatorname{Inf}(\pi)=\left\{o \in\right.$ Obs $\mid l_{i} \in \gamma(o)$ for infinitely many $i$ 's $\}$.

- Reachability and safety objectives. Given a set of observations $O \subseteq$ Obs, the reachability objective $\operatorname{Reach}(O)$ requires that an observation in $O$ is visited at least once, i.e. $\operatorname{Reach}(O)=\left\{\pi=\ell_{0} \sigma_{0} \ell_{1} \sigma_{1} \ldots \in\right.$ Play $\left.(G) \mid \exists k \geq 0 . \exists o \in O . \ell_{k} \in \gamma(o)\right\}$. Dually, the safety objective $\operatorname{Safe}(O)$ requires that only observations in $O$ be visited. Formally we have $\operatorname{Safe}(O)=\left\{\pi=\ell_{0} \sigma_{0} \ell_{1} \sigma_{1} \ldots \in \operatorname{Play}(G) \mid \forall k \geq 0 \cdot \exists o \in O: \ell_{k} \in\right.$ $\gamma(o)\}$.

- Büchi and coBüchi objectives. The Büchi objective Buchi $(O)$ requires that an observation in $O$ is visited infinitely often, i.e. $\operatorname{Buchi}(O)=\{\pi \mid$ $\operatorname{lnf}(\pi) \cap O \neq \emptyset\}$. Dually, the coBüchi objective coBuchi $(O)$ requires that only observations in $O$ be visited infinitely often. Formally we have $\operatorname{coBuchi}(O)=\{\pi \mid \operatorname{lnf}(\pi) \subseteq O\}$.

- Parity objectives. For $d \in \mathbb{N}$, let $p:$ Obs $\rightarrow\{0,1, \ldots, d\}$ be a priority function that maps each observation to a non-negative integer. The parity objective $\operatorname{Parity}(p)$ requires that the minimum priority that appears infinitely often is even. Formally we have $\operatorname{Parity}(p)=\{\pi \mid$ $\min \{p(O) \mid O \in \operatorname{Inf}(\pi)\}$ is even $\}$.

Observe that by definition for all objectives $\phi$, if $\pi \models \phi$ and $\operatorname{Obs}(\pi)=$ $\operatorname{Obs}\left(\pi^{\prime}\right)$, then $\pi^{\prime} \models \phi$.

Given a game structure of incomplete information $G$ and a state $\ell$ of $G$, we write $G_{\ell}$ for the game structure of incomplete information that results from $G$ by changing the initial state to $\ell$ : that is, if $G=\left\langle L, l_{0}, \Sigma, \Delta\right.$, Obs, $\left.\gamma\right\rangle$, then $G_{\ell}=\langle L, \ell, \Sigma, \Delta$, Obs, $\gamma\rangle$.

An event is a measurable set of paths (or a Borel objective), and given two strategies $\alpha$ and $\beta$, the probabilities of events are uniquely defined [21]. We denote by $\operatorname{Pr}_{\ell}^{\alpha, \beta}(\phi)$ the probability that an objective $\phi$ is satisfied in the game $G_{\ell}$ given strategies $\alpha$ and $\beta$. 
Sure winning and almost winning. A strategy $\lambda_{i}$ for Player $i$ in $G$ is sure-winning for an objective $\phi$ iff for all $\pi \in$ Outcome $_{i}\left(G, \lambda_{i}\right)$, we have $\pi \models \phi$. A strategy $\alpha$ for Player 1 is almost-winning for an objective $\phi$, if for all randomized strategies $\beta$ for Player 2 we have $\operatorname{Pr}_{l_{0}}^{\alpha, \beta}(\phi)=1$. The set of sure-winning (resp. almost-winning) states of a game structure of incomplete information $G$ for the objective $\phi$ is the set of states $\ell$ such that Player 1 has a deterministic sure-winning (resp. randomized almostwinning) observation-based strategy in $G_{\ell}$ for the objective $\phi$.

Theorem 1 (Determinacy [16]). For all Borel objectives $\phi$, for all complete-information game structures $G$ either there exists a deterministic sure-winning strategy for Player 1 for objective $\phi$, or there exists a deterministic sure-winning strategy for Player 2 for objective Play $(G) \backslash \phi$.

Notice that deterministic strategies suffice for sure-winning a game: given a randomized strategy $\alpha$ for Player 1 , let $\alpha^{D}$ be a deterministic strategy such that for all $\rho \in \operatorname{Pref}(G), \alpha^{D}(\rho)$ chooses an input letter from $\operatorname{Supp}(\alpha(\rho))$. Clearly Outcome ${ }_{1}\left(G, \alpha^{D}\right) \subseteq$ Outcome $_{1}(G, \alpha)$ and hence if $\alpha$ is sure-winning, then so is $\alpha^{D}$. The result also holds for observation-based strategies and for complete-information games. Hence deterministic strategies suffices for sure-winning and also for complete-information games randomized strategies are no more powerful than deterministic strategies. However, for almostwinning, randomized strategies are more powerful than deterministic strategies as shown by Example 1

Example 1 Consider the game structure shown in Figure 1. The observations $o_{1}, o_{2}, o_{3}, o_{4}$ are such that $\gamma\left(o_{1}\right)=\left\{\ell_{1}\right\}, \gamma\left(o_{2}\right)=\left\{\ell_{2}, \ell_{2}^{\prime}\right\}, \gamma\left(o_{3}\right)=$ $\left\{\ell_{3}, \ell_{3}^{\prime}\right\}, \gamma\left(o_{4}\right)=\left\{\ell_{4}\right\}$. The transitions are shown as labeled edges in the figure and the initial state is $\ell_{1}$. The objective of Player 1 is $\operatorname{Reach}\left(\left\{o_{4}\right\}\right)$, i.e. to reach state $\ell_{4}$. We argue that the game is not sure-winning for Player 1. Let $\alpha^{D}$ be any deterministic strategy for Player 1 . Consider a strategy $\beta$ for Player 2 as follows: for all $\rho \in \operatorname{Pref}(G)$ such that Last $(\rho) \in \gamma\left(o_{2}\right)$, if $\alpha^{D}(\rho)=a$, then in the previous round $\beta$ chooses the state $\ell_{2}$, and if $\alpha^{D}(\rho)=b$, then in the previous round $\beta$ chooses the state $\ell_{2}^{\prime}$. Given $\alpha^{D}$ and $\beta$, outcome $\left(G, \alpha^{D}, \beta\right)$ never reaches $\ell_{4}$. However we show that the game $G$ is almost-winning for Player 1 . Consider the randomized strategy that plays $a$ and $b$ uniformly at random at all states. Every time the game visits observation $o_{2}$, for any strategy for Player 2 , the game visits $\ell_{3}$ and $\ell_{3}^{\prime}$ with probability $\frac{1}{2}$, and hence also reaches $\ell_{4}$ with probability $\frac{1}{2}$. It easily follows that against all Player 2 strategies the play eventually reaches $\ell_{4}$ with probability 1. 


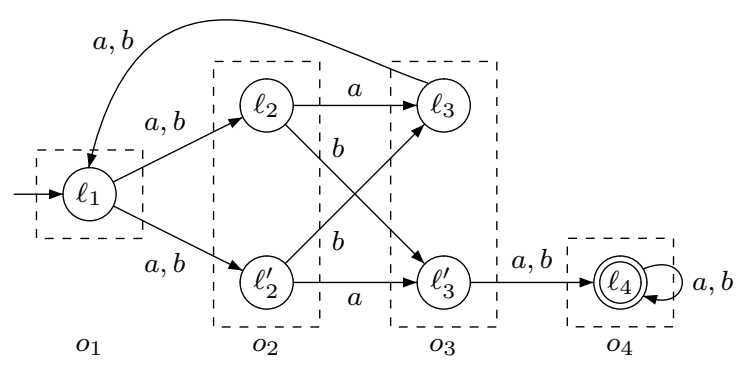

Figure 1: Game structure $G$.

Spoiling strategies. To spoil a strategy of Player 1 (for sure-winning), Player 2 does not need the full memory of the history of the play, he only needs counting strategies. We say that a deterministic strategy $\beta: \operatorname{Pref}(G) \times$ $\Sigma \rightarrow L$ for Player 2 is counting if for all prefixes $\rho, \rho^{\prime} \in \operatorname{Pref}(G)$ such that $|\rho|=\left|\rho^{\prime}\right|$ and $\operatorname{Last}(\rho)=\operatorname{Last}\left(\rho^{\prime}\right)$, and for all $\sigma \in \Sigma$, we have $\beta(\rho, \sigma)=$ $\beta\left(\rho^{\prime}, \sigma\right)$. Let $\mathcal{B}_{G}^{c}$ be the set of counting strategies for Player 2 . The memory needed by a counting strategy is only the number of turns that have been played. This type of strategy is sufficient to spoil the non-winning strategies of Player 1.

Proposition 1 Let $G$ be a game structure of incomplete information and $\phi$ be an objective. There exists an observation-based strategy $\alpha^{o} \in \mathcal{A}_{G}^{O}$ such that for all $\beta \in \mathcal{B}_{G}$ we have outcome $\left(G, \alpha^{o}, \beta\right) \in \phi$ if and only if there exists an observation-based strategy $\alpha^{o} \in \mathcal{A}_{G}^{O}$ such that for all counting strategies $\beta^{c} \in \mathcal{B}_{G}^{c}$ we have outcome $\left(G, \alpha^{o}, \beta^{c}\right) \in \phi$.

Proof. We prove the equivalent statement that: $\forall \alpha^{o} \in \mathcal{A}_{G}^{o} \cdot \exists \beta \in \mathcal{B}_{G}$ : outcome $\left(G, \alpha^{o}, \beta\right) \notin \phi$ iff $\forall \alpha^{o} \in \mathcal{A}_{G}^{o} \cdot \exists \beta^{c} \in \mathcal{B}_{G}^{c}$ : outcome $\left(G, \alpha^{o}, \beta^{c}\right) \notin \phi$. The right implication $(\leftarrow)$ is trivial. For the left implication $(\rightarrow)$, let $\alpha^{o} \in \mathcal{A}_{G}^{o}$ be an arbitrary observation-based strategy for Player 1 in $G$. Let $\beta \in \mathcal{B}_{G}$ be a strategy for Player 2 such that outcome $\left(G, \alpha^{o}, \beta\right) \notin \phi$. Let outcome $\left(G, \alpha^{o}, \beta\right)=\ell_{0} \sigma_{0} \ell_{1} \ldots \sigma_{n-1} \ell_{n} \sigma_{n} \ldots$ and define a counting strategy $\beta^{c}$ for Player 2 such that $\forall \rho \in \operatorname{Pref}(G) \cdot \forall \sigma \in \Sigma$ : if Last $(\rho)=\ell_{n-1}$ and $\sigma=\sigma_{n-1}$ for $n=|\rho|$, then $\beta^{c}(\rho, \sigma)=\ell_{n}$, and otherwise $\beta^{c}(\rho, \sigma)$ is fixed arbitrarily in the set $\operatorname{Post}_{\sigma}^{G}(\operatorname{Last}(\rho))$. Clearly, $\beta^{c}$ is a counting strategy and we have outcome $\left(G, \alpha^{o}, \beta\right)=\operatorname{outcome}\left(G, \alpha^{o}, \beta^{c}\right)$ and thus outcome $\left(G, \alpha^{o}, \beta^{c}\right) \notin \phi$. 


\section{Sure Winning}

First, we show that a game structure of incomplete information $G$ can be encoded in a game structure of complete information $G^{\mathrm{K}}$ such that for any Borel objective $\phi$, there exists a deterministic observation-based surewinning strategy for Player 1 in $G$ for $\phi$ if and only if there exists a deterministic sure-winning strategy for Player 1 in $G^{\mathrm{K}}$ for $\phi$. We obtain $G^{\mathrm{K}}$ using a subset construction. A state in $G^{\mathrm{K}}$ is a set of states of $G$ that corresponds to the knowledge of Player 1 . In the worst case, the size of $G^{\mathrm{K}}$ is exponentially larger than the size of $G$. Second, we present a fixed point algorithm based on antichains of set of states [10] whose correctness relies on our subset construction, but that avoids the explicit construction of $G^{\mathrm{K}}$.

\subsection{Subset construction for sure winning}

Subset construction. Given a game structure of incomplete information $G=\left(L, l_{0}, \Sigma, \Delta\right.$, Obs, $\left.\gamma\right)$, we define the knowledge-based subset construction of $G$ as the following game structure of complete information:

$$
G^{\mathrm{K}}=\left\langle\mathcal{L},\left\{l_{0}\right\}, \Sigma, \Delta^{\mathrm{K}}\right\rangle
$$

where $\mathcal{L}=2^{L} \backslash\{\emptyset\}$ and $\left(s_{1}, \sigma, s_{2}\right) \in \Delta^{\mathrm{K}}$ iff $\exists$ obs $\in$ Obs: $s_{2}=\operatorname{Post}_{\sigma}^{G}\left(s_{1}\right) \cap$ $\gamma($ obs $)$ and $s_{2} \neq \emptyset$. Notice that for all $s \in \mathcal{L}$, for all $\sigma \in \Sigma$, there exists $s^{\prime} \in \mathcal{L}:\left(s, \sigma, s^{\prime}\right) \in \Delta^{\mathrm{K}}$.

A (deterministic or randomized) strategy in $G^{\mathrm{K}}$ is called a knowledgebased strategy. To distinguish between a general strategy in $G$, an observation-based strategy in $G$ and a knowledge-based strategy in $G^{\mathrm{K}}$, we often use the notations $\alpha, \alpha^{o}$, and $\alpha^{\mathrm{K}}$ respectively.

Lemma 2 For all set $s \in \mathcal{L}$ that is reachable in $G^{\mathrm{K}}$, for all observation obs $\in$ Obs, we have either $s \subseteq \gamma$ (obs) or $s \cap \gamma($ obs $)=\emptyset$.

Proof. First, the property holds for $s=\left\{l_{0}\right\}$, the initial state in $G^{\mathrm{K}}$ as it is a singleton. Second, we show that the property holds for any successor $s^{\prime}$ of any state $s$ in $G^{\mathrm{K}}$. Assume that $\left(s, \sigma, s^{\prime}\right) \in \Delta^{\mathrm{K}}$. Then we know that $s^{\prime}=$ $\operatorname{Post}_{\sigma}^{G}(s) \cap \gamma($ obs $)$ for some obs $\in$ Obs. Hence, $s^{\prime} \subseteq \gamma($ obs $)$ and $s^{\prime} \cap \gamma\left(\right.$ obs $\left.^{\prime}\right)=\emptyset$ for any obs' $\neq$ obs since the set $\{\gamma($ obs $) \mid$ obs $\in$ Obs $\}$ partitions $L$.

By an abuse of notation, we define the observation of a play $\pi=s_{0} \sigma_{0} s_{1} \ldots \sigma_{n-1} s_{n} \sigma_{n} \cdots \in \operatorname{Play}\left(G^{\mathrm{K}}\right)$ as the sequence $\operatorname{Obs}(\pi)=$ $o_{0} \sigma_{0} o_{1} \ldots \sigma_{n-1} o_{n} \sigma_{n} \ldots$ such that for all $i \geq 0, s_{i} \subseteq \gamma\left(o_{i}\right)$. This sequence is 
unique by Lemma 2. We also say that $\pi$ satisfies an objective $\phi \subseteq(\text { Obs } \times \Sigma)^{\omega}$ iff $\operatorname{Obs}(\pi) \in \phi$. Lemma 2 justifies the following definition:

Definition 1 For a play $\pi=s_{0} \sigma_{0} s_{1} \ldots \sigma_{n-1} s_{n} \sigma_{n} \cdots \in \operatorname{Play}\left(G^{\mathrm{K}}\right)$, define the observation of $\pi$ as the unique sequence $\operatorname{Obs}(\pi)=o_{0} \sigma_{0} o_{1} \ldots \sigma_{n-1} o_{n} \sigma_{n} \ldots$ such that for any $i \geq 0, s_{i} \subseteq \gamma\left(o_{i}\right)$.

As above, we say that a play $\pi=s_{0} \sigma_{0} s_{1} \ldots \sigma_{n-1} s_{n} \sigma_{n} \cdots \in \operatorname{Play}\left(G^{\mathrm{K}}\right)$ satisfies an objective $\phi$ iff the sequence of observations $o_{0} o_{1} \ldots o_{n} \ldots$ such that for all $i \geq 0, \ell_{i} \in \gamma\left(o_{i}\right)$ belongs to $\phi$.

Lemma 3 If Player 1 has a deterministic sure-winning strategy in $G^{\mathrm{K}}$ for an objective $\phi$, then he has a deterministic observation-based sure-winning strategy in $G$ for $\phi$.

Proof. Let $\alpha^{\mathrm{K}}$ be a deterministic sure-winning strategy for Player 1 in $G^{\mathrm{K}}$ with the objective $\phi$. Define $\alpha^{o}$ a strategy for Player 1 in $G$ as follows: for every $\rho \in \operatorname{Pref}(G)$, let $\alpha^{o}(\rho)=\alpha^{\mathrm{K}}\left(\rho^{\mathrm{K}}\right)$ where $\rho^{\mathrm{K}}$ is defined from $\rho=\ell_{0} \sigma_{0} \ell_{1} \ldots \sigma_{n-1} \ell_{n}$ by $\rho^{\mathrm{K}}=s_{0} \sigma_{0} s_{1} \ldots \sigma_{n-1} s_{n}$ where $s_{i}=$ $\mathrm{K}\left(\mathrm{Obs}\left(\ell_{0} \sigma_{0} \ell_{1} \ldots \sigma_{i-1} \ell_{i}\right)\right)$ for each $0 \leq i \leq n$. Clearly, $\alpha^{o}$ is an deterministic observation-based strategy as $\operatorname{Obs}(\rho)=\operatorname{Obs}\left(\rho^{\prime}\right)$ implies $\rho^{\mathrm{K}}=\rho^{\prime \mathrm{K}}$.

By contradiction, assume that $\alpha^{o}$ is not a sure-winning strategy for Player 1 in $G$ with the objective $\phi$. Then there exists a play $\pi \in$ Outcome $_{1}\left(G, \alpha^{o}\right)$ such that $\pi \not \models \phi$. Let $\pi=\ell_{0} \sigma_{0} \ell_{1} \sigma_{1} \ldots$ and consider the infinite sequence $\pi^{\mathrm{K}}=s_{0} \sigma_{0} s_{1} \sigma_{1} \ldots$ where $s_{i}=\mathrm{K}(\mathrm{Obs}(\pi(i)))$ for each $i \geq 0$. We show that $\pi^{\mathrm{K}} \in$ Outcome $_{1}\left(G^{\mathrm{K}}, \alpha^{\mathrm{K}}\right)$. First, we have $s_{0}=\mathrm{K}(\operatorname{Obs}(\pi(0)))=\mathrm{K}\left(\operatorname{Obs}\left(\ell_{0}\right)\right)=\left\{\ell_{0}\right\}$. Second, for any $i \geq 0$, we have $s_{i}=\mathrm{K}(\operatorname{Obs}(\pi(i)))$ and by Lemma $1 s_{i+1}=\operatorname{Post}_{\sigma_{i}}^{G}\left(s_{i}\right) \cap \gamma($ obs $)$ where obs is such that $\ell_{i+1} \in \gamma($ obs $)$ and so $\left(s_{i}, \sigma_{i}, s_{i+1}\right) \in \Delta^{\mathrm{K}}$. Third, by definition of $\alpha^{o}$, we have $\sigma_{i}=\alpha^{o}(\pi(i))=\alpha^{\mathrm{K}}\left(\pi^{\mathrm{K}}(i)\right)$. This entails that $\pi^{\mathrm{K}} \in$ Outcome $_{1}\left(G^{\mathrm{K}}, \alpha^{\mathrm{K}}\right)$.

Now, observe that trivially $\ell_{i} \in \mathrm{K}(\mathrm{Obs}(\pi(i)))$ for any $i \geq 0$, that is $\ell_{i} \in s_{i}$ and so $s_{i} \cap \gamma\left(\right.$ obs $\left._{i}\right) \neq \emptyset$ where obs $i$ is the unique observation such that $\ell_{i} \in \gamma\left(\right.$ obs $\left._{i}\right)$. Hence, by Lemma 2, we have $s_{i} \subseteq \gamma\left(\right.$ obs $\left._{i}\right)$. Consequently, $\operatorname{Obs}\left(\pi^{\mathrm{K}}\right)=\operatorname{Obs}(\pi)$ and thus $\pi^{\mathrm{K}} \forall \phi$ which contradicts the fact that $\alpha^{\mathrm{K}}$ is a sure-winning strategy for Player 1 in $G^{\mathrm{K}}$ with the objective $\phi$. Therefore, $\alpha^{o}$ is a sure-winning strategy for Player 1 in $G$ with the objective $\phi$.

Lemma 4 If Player 1 does not have a deterministic sure-winning strategy in $G^{\mathrm{K}}$ for a Borel objective $\phi$, then Player 1 does not have a deterministic observation-based sure-winning strategy in $G$ for $\phi$. 
Proof. First, observe that if there exists no sure-winning strategy for Player 1 in $G^{\mathrm{K}}$ with objective $\phi$, then by the Determinacy Theorem (Theorem 1), Player 2 has a spoiling strategy $\beta^{S}$ such that for any play $\pi^{\mathrm{K}} \in$ Outcome $_{2}\left(G^{\mathrm{K}}, \beta^{S}\right)$ we have $\pi^{\mathrm{K}} \not \models \phi$. By contradiction, assume that Player 1 has an observation-based sure-winning strategy $\alpha^{o}$ in $G$ for $\phi$. We construct the tree $\mathrm{T}\left(\alpha^{o}, \beta^{S}\right)=\left\langle N, \Sigma, \Delta_{T}\right\rangle$ where $N=\bigcup_{i \geq 0} N_{i}$ and $\Delta_{T}=\bigcup_{i \geq 1} E_{i}$. The sets $N_{i}(i \geq 0)$ and $E_{i}(i \geq 1)$ are constructed inductively as follows: let $N_{0}=\left\{\ell_{0}\right\}$ and $s_{0}=\left\{\ell_{0}\right\}$. The following properties clearly hold for $i=0$ :

(a) $s_{i}=\left\{\operatorname{Last}(\rho) \mid \rho \in N_{i}\right\}$,

(b) $N_{i} \subseteq \operatorname{Pref}(G)$ and $s_{0} \sigma_{0} s_{1} \sigma_{1} \ldots \sigma_{i-1} s_{i} \in \operatorname{Pref}\left(G^{\mathrm{K}}\right)$,

(c) $\forall \rho, \rho^{\prime} \in N_{i}: \operatorname{Obs}(\rho)=\operatorname{Obs}\left(\rho^{\prime}\right)$ and

(d) $\forall \rho \in N_{i}: s_{i}=\mathrm{K}(\operatorname{Obs}(\rho))$.

Assume that we have constructed the sets $N_{i}$ and $s_{i}$ for $i=0, \ldots, k$ and the labels $\sigma_{i}$ for $i=0, \ldots, k-1$ such that $(a),(b),(c),(d)$ hold and let us construct $N_{k+1}, s_{k+1}$ and $\sigma_{k}$ such that $(a),(b),(c),(d)$ hold for $i=k+1$. Let

- $\rho \in N_{k}$ and $\tau=\operatorname{Obs}(\rho)$,

- $\sigma_{k}=\alpha^{o}(\rho)$ (by $(c), \sigma_{k}$ is independent of the choice of $\rho$ since $\alpha^{o}$ is observation-based),

- $s_{k+1}=\beta^{S}\left(s_{0} \sigma_{0} s_{1} \sigma_{1} \ldots \sigma_{k-1} s_{k}, \sigma_{k}\right)$,

- $N_{k+1}=\left\{\rho \sigma_{k} l^{\prime} \mid \rho \in N_{k} \wedge l^{\prime} \in s_{k+1} \wedge\left(\operatorname{Last}(\rho), \sigma, l^{\prime}\right) \in \Delta\right\}$,

- $E_{k+1}=\left\{\left(\rho, \sigma_{k}, \rho \sigma_{k} l^{\prime}\right) \mid \rho \in N_{k} \wedge \rho \sigma_{k} l^{\prime} \in N_{k+1}\right\}$.

Then, we have $(i) s_{k+1} \neq \emptyset(i i)\left(s_{k}, \sigma_{k}, s_{k+1}\right) \in \Delta^{\mathrm{K}}$ and thus $s_{k+1} \subseteq$ Post $_{\sigma_{k}}^{G}\left(s_{k}\right)$ and $($ iii $) s_{k+1} \subseteq \gamma($ obs $)$ for some obs $\in$ Obs. We prove that:

$\left(a^{\prime}\right) s_{k+1}=\left\{\operatorname{Last}(\rho) \mid \rho \in N_{k+1}\right\}$. By definition of $N_{k+1}$, we have $\forall \rho \in$ $N_{k+1}: \operatorname{Last}(\rho) \in s_{k+1}$. By $(i i)$, we have that $\forall l^{\prime} \in s_{k+1} \cdot \exists l \in s_{k}$ : $\left(l, \sigma_{k}, l^{\prime}\right) \in \Delta$ and by $(a)$, this entails that $\forall l^{\prime} \in s_{k+1} \cdot \exists \rho \in N_{k}$ : $\rho \sigma_{k} l^{\prime} \in N_{k+1}$, that is $s_{k+1} \subseteq\left\{\operatorname{Last}(\rho) \mid \rho \in N_{k}\right\}$;

$\left(b^{\prime}\right) N_{k+1} \subseteq \operatorname{Pref}(G)$ and $s_{0} \sigma_{0} \ldots \sigma_{k} s_{k+1} \in \operatorname{Pref}\left(G^{\mathrm{K}}\right)$. This follows from $(b)$ and the definition of $N_{k+1}$ and $s_{k+1}$; 
$\left(c^{\prime}\right) \forall \rho, \rho^{\prime} \in N_{k+1}: \operatorname{Obs}(\rho)=\operatorname{Obs}\left(\rho^{\prime}\right)$. Let $\rho, \rho^{\prime} \in N_{k+1}$. By definition of $N_{k+1}$ and by (iii), we have $\operatorname{Obs}(\rho)=\tau \sigma_{k}$ obs where $\tau$ is independent of $\rho$ by $(c)$. Therefore $\operatorname{Obs}\left(\rho^{\prime}\right)=\tau \sigma_{k}$ obs;

$\left(d^{\prime}\right) \forall \rho \in N_{k+1}: s_{k+1}=\mathrm{K}(\mathrm{Obs}(\rho))$. This is established by Lemma 1 .

Now, by $(a)$ and $(i)$, the tree $\mathbf{T}\left(\alpha^{o}, \beta^{S}\right)$ is infinite and by König's Lemma it contains an infinite sequence $\rho_{0}, \sigma_{0}, \rho_{1}, \sigma_{1}, \ldots$ such that $\rho_{0}=\left\{\ell_{0}\right\}$ and for all $i \geq 1,\left(\rho_{i-1}, \sigma_{i-1}, \rho_{i}\right) \in \Delta_{T}$. Correspondingly, let $\pi=\ell_{0} \sigma_{0} \ell_{1} \sigma_{1} \ldots$ such that $\ell_{i}=\operatorname{Last}\left(\rho_{i}\right)$ for each $i \geq 0$. By construction of $\mathrm{T}\left(\alpha^{o}, \beta^{S}\right)$, we have $\pi(i) \in \operatorname{Pref}(G)$ and $\sigma_{i}=\alpha^{o}(\pi(i))$ for all $i \geq 0$. Hence $\pi \in$ Outcome $_{1}\left(G, \alpha^{o}\right)$ and therefore $\pi \models \phi$. Consider the infinite sequence $\pi^{\mathrm{K}}=s_{0} \sigma_{0} s_{1} \sigma_{1} \ldots$ (the set $s_{i}$ have been constructed together with the sets $N_{i}$ ). By construction of $\mathrm{T}\left(\alpha^{o}, \beta^{S}\right)$, we have $s_{i+1}=\beta^{S}\left(s_{0} \sigma_{0} \ldots \sigma_{i-1} s_{i}, \sigma_{i}\right)$ for all $i \geq 0$ and thus $\pi^{\mathrm{K}} \in$ Outcome $_{2}\left(G^{\mathrm{K}}, \beta^{S}\right)$ and $\pi^{\mathrm{K}} \not \neq \phi$. However, by $(a)$ and $(d)$ we have $\operatorname{Obs}(\pi)=\operatorname{Obs}\left(\pi^{\mathrm{K}}\right)$ which contradicts the fact that $\pi \models \phi$ and $\pi^{\mathrm{K}} \not \models \phi$. Therefore, Player 1 cannot have a sure-winning observation-based strategy $\alpha^{o}$ in $G$ for $\phi$.

Lemma 3 and Lemma 4 yield Theorem 2.

Theorem 2 Player 1 has a deterministic sure-winning strategy in $G^{\mathrm{K}}$ for a Borel objective $\phi$ if and only if Player 1 has a deterministic observationbased sure-winning strategy in $G$ for $\phi$.

\subsection{Two interpretations of the $\mu$-calculus}

Form the results of Section 3.1, we could solve a game $G$ of incomplete information with objective $\phi$ by constructing the knowledge-based subset construction $G^{\mathrm{K}}$ of $G$ and solve the resulting game of complete information for the objective $\phi$ using standard methods. For the important class of $\omega$ regular objectives, there exists a fixed point theory -the $\mu$-calculus- for this purpose [8]. When run on $G^{\mathrm{K}}$, those fixed point algorithms compute sets of sets of states of the game $G$. An important property of those sets is that they are downward closed for set inclusion: if Player 1 has a deterministic strategy to win the game $G$ when her knowledge is the set $s$, then she also has a deterministic strategy to win the game when her knowledge is $s^{\prime}$ with $s^{\prime} \subseteq s$. And thus, if $s$ is a sure-winning state of $G^{\mathrm{K}}$, then so is $s^{\prime}$. Based on this property, we devise a new algorithm for solving games of incomplete information.

An antichain of nonempty sets of states is a set $q \subseteq 2^{L} \backslash \emptyset$ such that for all $s, s^{\prime} \in q$, we have $s \not \subset s^{\prime}$. Let $\mathcal{A}$ be the set of antichains of nonempty subsets 
of $L$ and consider the following partial order on $\mathcal{A}$ : for all $q, q^{\prime} \in \mathcal{A}$, let $q \sqsubseteq q^{\prime}$ iff $\forall s \in q: \exists s^{\prime} \in q^{\prime}: s \subseteq s^{\prime}$. For $q \subseteq 2^{L} \backslash \emptyset$, define the set of maximal elements of $q$ by $\lceil q\rceil=\left\{s \in q \mid s \neq \emptyset \wedge \nexists s^{\prime} \in q: s \subset s^{\prime}\right\}$. Clearly, $\lceil q\rceil$ is an antichain. The least upper bound of $q, q^{\prime} \in \mathcal{A}$ is $q \sqcup q^{\prime}=\left\lceil\left\{s \mid s \in q \vee s \in q^{\prime}\right\}\right\rceil$ and their greatest lower bound is $q \sqcap q^{\prime}=\left\lceil\left\{s \cap s^{\prime} \mid s \in q \wedge s^{\prime} \in q^{\prime}\right\}\right\rceil$. The definition of those two operators extends naturally to sets of antichains, and the greatest element of $\mathcal{A}$ is $T=\{L\}$ and the least element is $\perp=\emptyset$. The partially ordered set $\langle\mathcal{A}, \sqsubseteq, \sqcup, \sqcap, \top, \perp\rangle$ forms a complete lattice. We see antichains of state sets as a symbolic representation of $\subseteq$-downward closed sets of state sets.

A game-lattice is a complete lattice $V$ together with a predecessor operator CPre $: V \rightarrow V$. Given a game structure $G=\left\langle L, l_{0}, \Sigma, \Delta\right.$, Obs, $\left.\gamma\right\rangle$ and its knowledge-based subset construction $G^{\mathrm{K}}=\left\langle\mathcal{L},\left\{l_{0}\right\}, \Sigma, \Delta^{\mathrm{K}}\right\rangle$, we consider two game-lattices: the lattice of subsets $\langle\mathcal{S}, \subseteq, \cup, \cap, \mathcal{L}, \emptyset\rangle$ (where $\mathcal{S}=2^{\mathcal{L}}$ ) with the predecessor operator CPre $: \mathcal{S} \rightarrow \mathcal{S}$ defined by:

$$
\operatorname{CPre}(q)=\left\{s \in \mathcal{L} \mid \exists \sigma \in \Sigma \cdot \forall s^{\prime}: \text { if }\left(s, \sigma, s^{\prime}\right) \in \Delta^{\mathrm{K}} \text { then } s^{\prime} \in q\right\}
$$

and the lattice of antichains $\langle\mathcal{A}, \sqsubseteq, \sqcup, \sqcap,\{L\}, \emptyset\rangle$ with the predecessor operator $\lceil$ CPre $\rceil: \mathcal{A} \rightarrow \mathcal{A}$ defined by:

$$
\lceil\operatorname{CPre}\rceil(q)=\left\lceil\left\{s \in \mathcal{L} \mid \exists \sigma \in \Sigma \cdot \forall o \in \text { Obs } \cdot \exists s^{\prime} \in q: \operatorname{Post}_{\sigma}(s) \cap \gamma(o) \subseteq s^{\prime}\right\}\right\rceil
$$

The $\mu$-calculus formulas are generated by the grammar:

$$
\varphi::=\text { obs }|x| \varphi \vee \varphi|\varphi \wedge \varphi| \operatorname{pre}(\varphi)|\mu x . \varphi| \nu x . \varphi
$$

for atomic propositions obs $\in$ Obs and variables $x$. We can define $\neg$ obs as a shortcut for $\bigvee_{o^{\prime} \in \mathrm{Obs} \backslash\{o\}} o^{\prime}$. A variable is free in a formula $\varphi$ if it is not under the scope of a quantification $\mu x$ or $\nu x$. A formula $\varphi$ is closed if it contains no free variable. Given a game-lattice $V$, a valuation $\mathcal{E}$ for the variables is a function that maps every variable $x$ to an element in $V$. For $q \in V$, we write $\mathcal{E}[x \mapsto q]$ for the valuation that agrees with $\mathcal{E}$ on all variables, except that $x$ is mapped to $q$. Given a game-lattice $V$, and a valuation $\mathcal{E}$, each $\mu$-calculus formula $\varphi$ specifies an element $\llbracket \varphi \rrbracket_{\mathcal{E}}^{V}$ of $V$, which is defined inductively by the following equations: 


\begin{tabular}{|c|}
\hline Lattice of subs \\
\hline$\llbracket$ obs $\rrbracket_{\mathcal{E}}^{\mathcal{S}}=\{s \in \mathcal{L} \mid s \subseteq \gamma($ obs $)\}$ \\
\hline$\llbracket x \rrbracket_{\mathcal{E}}^{\mathcal{S}}=\mathcal{E}(x)$ \\
\hline$\llbracket \varphi_{1}\left\{\begin{array}{c}\vee \\
\wedge\end{array}\right\} \varphi_{2} \rrbracket_{\mathcal{E}}^{\mathcal{S}}=\llbracket \varphi_{1} \rrbracket_{\mathcal{E}}^{\mathcal{S}}\left\{\begin{array}{c}\cup \\
\bigcap\end{array}\right\} \llbracket \varphi_{2} \rrbracket_{\mathcal{E}}^{\mathcal{S}}$ \\
\hline$\llbracket p r e(\varphi) \rrbracket_{\mathcal{E}}=\operatorname{CPre}\left(\llbracket \varphi \rrbracket_{\mathcal{E}}^{\mathcal{S}}\right)$ \\
\hline$\llbracket\left\{\begin{array}{l}\mu \\
\nu\end{array}\right\} x \cdot \varphi \rrbracket_{\mathcal{E}}^{\mathcal{S}}=\left\{\begin{array}{l}\cap \\
\cup\end{array}\right\}\left\{q \mid q=\llbracket \varphi \rrbracket_{\mathcal{E}[x \mapsto q]}^{\mathcal{S}}\right\}$ \\
\hline Latti \\
\hline$\llbracket$ obs $\rrbracket_{\mathcal{E}}^{\mathcal{A}}=\{\gamma($ obs $)\}$ \\
\hline$\llbracket x \rrbracket_{\mathcal{E}}^{\mathcal{A}}=\mathcal{E}(x)$ \\
\hline$\llbracket \varphi_{1}\left\{\begin{array}{c}\vee \\
\wedge\end{array}\right\} \varphi_{2} \rrbracket_{\mathcal{E}}^{\mathcal{A}}=\llbracket \varphi_{1} \rrbracket_{\mathcal{E}}^{\mathcal{A}}\left\{\begin{array}{c}\sqcup \\
\square\end{array}\right\} \llbracket \varphi_{2} \rrbracket_{\mathcal{E}}^{\mathcal{A}}$ \\
\hline$\llbracket p r e(\varphi) \rrbracket_{\mathcal{E}}^{\mathcal{A}}=\lceil\operatorname{CPre}\rceil\left(\llbracket \varphi \rrbracket_{\mathcal{E}}^{\mathcal{A}}\right)$ \\
\hline$\llbracket\left\{\begin{array}{c}\mu \\
\nu\end{array}\right\} x \cdot \varphi \rrbracket_{\mathcal{E}}^{\mathcal{A}}=\left\{\begin{array}{l}\square \\
\sqcup\end{array}\right\}\left\{q \mid q=\llbracket \varphi \rrbracket_{\mathcal{E}[x \mapsto q]}^{\mathcal{A}}\right\}$ \\
\hline
\end{tabular}

If $\varphi$ is a closed formula, then $\llbracket \varphi \rrbracket^{V}=\llbracket \varphi \rrbracket_{\mathcal{E}}^{V}$ for any valuation $\mathcal{E}$.

Theorem $3([8])$ For all $\omega$-regular objectives $\phi$ there exists a closed $\mu$ calculus formula $\varphi_{\phi}$ such that for all complete information game structures $G, \llbracket \varphi_{\phi} \rrbracket^{\mathcal{S}}$ is the set of sure-winning states of $G$ for $\phi$.

Downward closure. Given a set $q \in \mathcal{S}$, the downward closure of $q$ is the set $q \downarrow=\left\{s \in \mathcal{L} \mid \exists s^{\prime} \in q: s \subseteq s^{\prime}\right\}$. Observe that in particular, for all $q \in \mathcal{S}$ we have $\emptyset \notin q \downarrow$ and $\lceil q\rceil \downarrow=q \downarrow$. The sets $q \downarrow(q \in \mathcal{S})$ are the downward closed sets. We say that a valuation $\mathcal{E}$ for the variables is downward closed if every variable $x$ is mapped to a downward closed set, that is $\mathcal{E}(x)=\mathcal{E}(x) \downarrow$.

Lemma 5 For all downward closed sets $q, q^{\prime} \in \mathcal{S}$, we have

$$
\left\lceil q \cap q^{\prime}\right\rceil=\lceil q\rceil \sqcap\left\lceil q^{\prime}\right\rceil \quad \text { and } \quad\left\lceil q \cup q^{\prime}\right\rceil=\lceil q\rceil \sqcup\left\lceil q^{\prime}\right\rceil .
$$

Lemma 6 For all $\mu$-calculus formulas $\varphi$, for all downward closed valuations $\mathcal{E}$ in the lattice of subsets, the set $\llbracket \varphi \rrbracket_{\mathcal{E}}^{\mathcal{S}}$ is downward closed.

Proof. We prove this lemma by induction on the structure of $\varphi$.

- if $\varphi \equiv$ obs for obs $\in$ Obs. It is immediate to show that $\llbracket \varphi \rrbracket_{\mathcal{E}}^{\mathcal{S}}=\llbracket \varphi \rrbracket_{\mathcal{E}}^{\mathcal{S}}$. 
- if $\varphi \equiv x$ for a variable $x$. We have $\llbracket \varphi \rrbracket_{\mathcal{E}}^{\mathcal{S}}=\mathcal{E}(x)$ which is downward closed by hypothesis.

- if $\varphi \equiv \varphi_{1}\left\{\begin{array}{c}\vee \\ \wedge\end{array}\right\} \varphi_{2}$ and both $\llbracket \varphi_{1} \rrbracket_{\mathcal{E}}^{\mathcal{S}}$ and $\llbracket \varphi_{2} \rrbracket_{\mathcal{E}}^{\mathcal{S}}$ are downward closed. Then we have $\llbracket \varphi \rrbracket_{\mathcal{E}}^{\mathcal{S}}=\llbracket \varphi_{1} \rrbracket_{\mathcal{E}}\left\{\begin{array}{c}\cup \\ \cap\end{array}\right\} \llbracket \varphi_{2} \rrbracket_{\mathcal{E}}^{\mathcal{S}}$ and the result follows from the fact that union and intersection of downward closed sets are downward closed.

- if $\varphi \equiv \operatorname{pre}\left(\varphi_{1}\right)$ and $\llbracket \varphi_{1} \rrbracket_{\mathcal{E}}$ is downward closed. We show that $\llbracket \varphi \rrbracket_{\mathcal{E}}^{\mathcal{S}}$ is downward closed. Let $s_{1} \in \llbracket \varphi \rrbracket_{\mathcal{E}}^{\mathcal{S}}$ and let $s_{2} \in \mathcal{L}$ such that $s_{2} \subseteq s_{1}$. Let us show that $s_{2} \in \llbracket \varphi \rrbracket_{\mathcal{E}}^{\mathcal{S}}$. By definition of CPre, since $s_{1} \in \llbracket$ pre $\left(\varphi_{1}\right) \rrbracket_{\mathcal{E}}^{\mathcal{S}}$, there exists $\sigma \in \Sigma$ such that for any $s_{1}^{\prime}$, if $\left(s_{1}, \sigma, s_{1}^{\prime}\right) \in \Delta^{\mathrm{K}}$ then $s_{1}^{\prime} \in \llbracket \varphi_{1} \rrbracket_{\mathcal{E}}^{\mathcal{S}}$. Consider any $s_{2}^{\prime}$ such that $\left(s_{2}, \sigma, s_{2}^{\prime}\right) \in \Delta^{\mathrm{K}}$. According to Definition 1, we have $s_{2}^{\prime}=\operatorname{Post}_{\sigma}\left(s_{2}\right) \cap \gamma$ (obs) $\neq \emptyset$ for some obs $\in$ Obs. Now, let $s_{1}^{\prime}=\operatorname{Post}_{\sigma}\left(s_{1}\right) \cap \gamma($ obs $)$. Since $s_{2} \subseteq s_{1}$, we have $s_{2}^{\prime} \subseteq s_{1}^{\prime}$ and thus $s_{1}^{\prime} \neq \emptyset$. Therefore $\left(s_{1}, \sigma, s_{1}^{\prime}\right) \in \Delta^{\mathrm{K}}$ and so $s_{1}^{\prime} \in \llbracket \varphi_{1} \rrbracket_{\mathcal{E}}^{\mathcal{S}}$. As the latter set is downward closed, we also have $s_{2}^{\prime} \in \llbracket \varphi_{1} \rrbracket_{\mathcal{E}}^{\mathcal{S}}$ and thus $s_{2} \in \llbracket \operatorname{pre}\left(\varphi_{1}\right) \rrbracket_{\mathcal{E}}$.

- if $\varphi \equiv \nu x . \varphi_{1}$ and $\llbracket \varphi_{1} \rrbracket_{\mathcal{E}^{\prime}}^{\mathcal{S}}$ is downward closed for any downward closed valuation $\mathcal{E}^{\prime}$. By Tarski's theorem, $\llbracket \varphi \rrbracket_{\mathcal{E}}^{\mathcal{S}}$ is one of the set in the infinite sequence $q_{0}, q_{1}, \ldots$ defined by $q_{0}=\mathcal{L}$ and for every $i \geq 1$, $q_{i}=\llbracket \varphi \rrbracket_{\mathcal{E}\left[x \mapsto q_{i-1}\right]}^{\mathcal{S}}$. Since $q_{0}$ is downward closed, every $q_{i}(i \geq 1)$ is also downward closed by the induction hypothesis.

- if $\varphi \equiv \mu x \cdot \varphi_{1}$ and $\llbracket \varphi_{1} \rrbracket_{\mathcal{E}^{\prime}}^{\mathcal{S}}$ is downward closed for any downward closed valuation $\mathcal{E}^{\prime}$. The proof is similar to the previous case.

Lemma 7 For all $\mu$-calculus formulas $\varphi$, for all downward closed valuations $\mathcal{E}$ in the lattice of subsets, we have $\left\lceil\llbracket \varphi \rrbracket_{\mathcal{E}}^{\mathcal{S}}\right\rceil=\llbracket \varphi \rrbracket_{\lceil\mathcal{E}\rceil}^{\mathcal{A}}$ where $\lceil\mathcal{E}\rceil$ is a valuation in the lattice of antichains defined by $\lceil\mathcal{E}\rceil(x)=\lceil\mathcal{E}(x)\rceil$ for all variables $x$.

Proof. We prove this by induction on the structure of $\varphi$.

- if $\varphi \equiv$ obs for obs $\in$ Obs. The claim is immediate.

- if $\varphi \equiv x$ for a variable $x$. We have $\left\lceil\llbracket \varphi \rrbracket_{\mathcal{E}}^{\mathcal{S}}\right\rceil=\lceil\mathcal{E}(x)\rceil=\llbracket \varphi \rrbracket_{\lceil\mathcal{E}\rceil}^{\mathcal{A}}$. 
- if $\varphi \equiv \varphi_{1}\left\{\begin{array}{c}\vee \\ \wedge\end{array}\right\} \varphi_{2}$ and both $\left\lceil\llbracket \varphi_{1} \rrbracket_{\mathcal{E}}^{\mathcal{S}}\right\rceil=\llbracket \varphi_{1} \rrbracket_{\lceil\mathcal{E}\rceil}^{\mathcal{A}}$ and $\left\lceil\llbracket \varphi_{2} \rrbracket_{\mathcal{E}}^{\mathcal{S}}\right\rceil=\llbracket \varphi_{2} \rrbracket_{\lceil\mathcal{E}\rceil}^{\mathcal{A}}$.

Using Lemma 5 and Lemma 6, we have successively:

$$
\begin{aligned}
& \left\lceil\llbracket \varphi \rrbracket_{\mathcal{E}}^{\mathcal{S}}\right\rceil=\left\lceil\llbracket \varphi_{1} \rrbracket_{\mathcal{E}}^{\mathcal{S}}\left\{\begin{array}{c}
\cup \\
\cap
\end{array}\right\} \llbracket \varphi_{2} \rrbracket_{\mathcal{E}}^{\mathcal{S}}\right\rceil= \\
& \left\lceil\llbracket \varphi_{1} \rrbracket_{\mathcal{E}}^{\mathcal{S}}\right\rceil\left\{\begin{array}{c}
\sqcup \\
\square
\end{array}\right\}\left\lceil\llbracket \varphi_{2} \rrbracket_{\mathcal{E}}^{\mathcal{S}}\right\rceil=\llbracket \varphi_{1} \rrbracket_{\lceil\mathcal{E}\rceil}^{\mathcal{A}\rceil}\left\{\begin{array}{c}
\sqcup \\
\square
\end{array}\right\} \llbracket \varphi_{2} \rrbracket_{\lceil\mathcal{E}\rceil}^{\mathcal{A}\rceil} .
\end{aligned}
$$

- if $\varphi \equiv \operatorname{pre}\left(\varphi_{1}\right)$ and $\left\lceil\llbracket \varphi_{1} \rrbracket_{\mathcal{E}}^{\mathcal{S}}\right\rceil=\llbracket \varphi_{1} \rrbracket_{\lceil\mathcal{E}\rceil}^{\mathcal{A}}$.

1. We prove the inclusion $\llbracket \varphi \rrbracket_{[\mathcal{E}\rceil}^{\mathcal{A}} \subseteq\left\lceil\llbracket \varphi \rrbracket_{\mathcal{E}}^{\mathcal{S}}\right\rceil$. First, we show that for any state $s \in\lceil\operatorname{CPre}\rceil\left(\llbracket \varphi_{1} \rrbracket_{\lceil\mathcal{E}\rceil}^{\mathcal{A}}\right)$, we have $s \in \operatorname{CPre}\left(\llbracket \varphi_{1} \rrbracket_{\mathcal{E}}^{\mathcal{S}}\right)$. We know that there exists $\sigma \in \Sigma$ such that $\forall o \in$ Obs $\cdot \exists s^{\prime \prime} \in \llbracket \varphi_{1} \rrbracket_{\lceil\mathcal{E}\rceil}^{\mathcal{A}}$ : Post $_{\sigma}(s) \cap \gamma(o) \subseteq s^{\prime \prime}$. Since $\left\lceil\llbracket \varphi_{1} \rrbracket_{\mathcal{E}}^{\mathcal{S}}\right\rceil=\llbracket \varphi_{1} \rrbracket_{\lceil\mathcal{E}\rceil}^{\mathcal{A}}$ (induction hypothesis), it is clear that for such $\sigma$, if $\left(s, \sigma, s^{\prime}\right) \in \Delta^{\mathrm{K}}$, then there exists $s^{\prime \prime} \in\left\lceil\llbracket \varphi_{1} \rrbracket_{\mathcal{E}} \mathcal{E}_{\mathcal{E}}\right\rceil$ such that $s^{\prime} \subseteq s^{\prime \prime}$. And since $\llbracket \varphi_{1} \rrbracket_{\mathcal{E}}$ is downward closed (by Lemma 6) we have $s^{\prime} \in \llbracket \varphi_{1} \rrbracket_{\mathcal{E}}$, so that $s \in \operatorname{CPre}\left(\llbracket \varphi_{1} \rrbracket_{\mathcal{E}}^{\mathcal{S}}\right)$ (and thus $s \in \llbracket \varphi \rrbracket_{\mathcal{E}}^{\mathcal{S}}$ ).

Second, we show that $s$ is maximal in $\llbracket \varphi \rrbracket_{\mathcal{E}}^{\mathcal{S}}$. By contradiction, assume that there exists $s_{1} \in \llbracket \varphi \rrbracket_{\mathcal{E}}^{\mathcal{S}}$ with $s \subset s_{1}$. Then, by the same argument as in the first part of the proof of the inclusion $\left\lceil\llbracket \varphi \rrbracket_{\mathcal{E}}^{\mathcal{S}}\right\rceil \subseteq \llbracket \varphi \rrbracket_{\lceil\mathcal{E}\rceil}^{\mathcal{A}}$, we have that $s_{1}$ satisfies the definition of $\lceil\operatorname{CPre}\rceil\left(\llbracket \varphi_{1} \rrbracket_{\lceil\mathcal{E}\rceil}^{\mathcal{A}\rceil}\right)$ up to the operator $\lceil\cdot\rceil$. This means that $s$ is not maximal in $\lceil\operatorname{CPre}\rceil\left(\llbracket \varphi_{1} \rrbracket_{\lceil\mathcal{E}\rceil}^{\mathcal{A}}\right)$, a contradiction.

2. We prove the inclusion $\left\lceil\llbracket \varphi \rrbracket_{\mathcal{E}}^{\mathcal{S}}\right\rceil \subseteq \llbracket \varphi \rrbracket_{\lceil\mathcal{E}\rceil}^{\mathcal{A}}$. This is trivial if $\left\lceil\llbracket \varphi \rrbracket_{\mathcal{E}}^{\mathcal{S}}\right\rceil=$ $\emptyset$. Otherwise, let us first show that $\llbracket \varphi_{1} \rrbracket_{\lceil\mathcal{E}\rceil}^{\mathcal{A}} \neq \emptyset$. Let $s \in\left\lceil\llbracket \varphi \rrbracket_{\mathcal{E}}^{\mathcal{S}}\right\rceil$. Then, there exists $\sigma \in \Sigma$ such that for any $s^{\prime}$, if $\left(s, \sigma, s^{\prime}\right) \in \Delta^{\mathrm{K}}$ then $s^{\prime} \in \llbracket \varphi_{1} \rrbracket_{\mathcal{E}}^{\mathcal{S}}$. Since the transition relation of $G$ is total and the observations partition the state space, we have $\operatorname{Post}_{\sigma}(s) \cap \gamma($ obs $) \neq$ $\emptyset$ for some obs $\in$ Obs. Therefore, $\llbracket \varphi_{1} \rrbracket_{\mathcal{E}}^{\mathcal{S}}$ is nonempty and so is $\llbracket \varphi_{1} \rrbracket_{\lceil\mathcal{E}\rceil}^{\mathcal{A}}$.

Now, we proceed with the proof of inclusion. Let $s \in\left\lceil\llbracket \varphi \rrbracket_{\mathcal{E}}^{\mathcal{S}}\right\rceil$, and let $\sigma \in \Sigma$ such that such that for any $s^{\prime}$, if $\left(s, \sigma, s^{\prime}\right) \in \Delta^{\mathrm{K}}$ then $s^{\prime} \in \llbracket \varphi_{1} \rrbracket_{\mathcal{E}}^{\mathcal{S}}$. Let us show that $s \in\lceil\operatorname{CPre}\rceil\left(\llbracket \varphi_{1} \rrbracket_{\lceil\mathcal{E}\rceil}^{\mathcal{A}}\right)$. First, consider an arbitrary observation obs $\in$ Obs and let $s^{\prime}=\operatorname{Post}_{\sigma}(s) \cap \gamma($ obs $)$. We must show that there exists $s^{\prime \prime} \in \llbracket \varphi_{1} \rrbracket_{\lceil\mathcal{E}\rceil}^{\mathcal{A}\rceil}$ such that $s^{\prime} \subseteq s^{\prime \prime}$. This is obvious if $s^{\prime}=\emptyset$ since $\llbracket \varphi_{1} \rrbracket_{\lceil\mathcal{E}\rceil}^{\mathcal{A}\rceil}$ is nonempty. Otherwise, by Definition 1 we have $\left(s, \sigma, s^{\prime}\right) \in \Delta^{\mathrm{K}}$ and therefore $s^{\prime} \in \llbracket \varphi_{1} \rrbracket_{\mathcal{E}}^{\mathcal{S}}$. Since 
$\llbracket \varphi_{1} \rrbracket_{\lceil\mathcal{E}\rceil}^{\mathcal{A}}=\left\lceil\llbracket \varphi_{1} \rrbracket_{\mathcal{E}}^{\mathcal{S}}\right\rceil$ (induction hypothesis), there exists $s^{\prime \prime} \in \llbracket \varphi_{1} \rrbracket_{\lceil\mathcal{E}\rceil}^{\mathcal{A}}$ such that $s^{\prime} \subseteq s^{\prime \prime}$.

Second, let us show that $s$ is maximal in $\lceil\operatorname{CPre}\rceil\left(\llbracket \varphi_{1} \rrbracket_{\lceil\mathcal{E}\rceil}^{\mathcal{A}}\right)$. By contradiction, assume that there exists $s_{1} \in\lceil\mathrm{CPre}\rceil\left(\llbracket \varphi_{1} \rrbracket_{\lceil\mathcal{E}\rceil}^{\mathcal{A}\rceil}\right)$ with $s \subset s_{1}$. Then, by the same argument as in the first part of the proof of the inclusion $\llbracket \varphi \rrbracket_{\lceil\mathcal{E}\rceil}^{\mathcal{A}\rceil} \subseteq\left\lceil\llbracket \varphi \rrbracket_{\mathcal{E}}^{\mathcal{S}}\right\rceil$, we have $s_{1} \in \llbracket \varphi \rrbracket_{\mathcal{E}}^{\mathcal{S}}$. This implies that $s \notin\left\lceil\llbracket \varphi \rrbracket_{\mathcal{E}}^{\mathcal{S}}\right\rceil$, a contradiction.

- if $\varphi \equiv \nu x . \varphi_{1}$ and $\left\lceil\llbracket \varphi_{1} \rrbracket_{\mathcal{E}^{\prime}}^{\mathcal{S}}\right\rceil=\llbracket \varphi_{1} \rrbracket_{\left.\digamma_{\mathcal{E}^{\prime}}\right\rceil}^{\mathcal{A}}$ for any downward closed valuation $\mathcal{E}^{\prime}$. By Tarski's theorem, $\llbracket \varphi \rrbracket_{\mathcal{E}}^{\mathcal{S}}$ is one of the set in the infinite sequence $q_{0}, q_{1}, \ldots$ defined by $q_{0}=\mathcal{L}$ and for every $i \geq 1, q_{i}=\llbracket \varphi \rrbracket_{\mathcal{E}\left[x \mapsto q_{i-1}\right]}^{\mathcal{S}} ;$ and similarly, $\llbracket \varphi \rrbracket_{\lceil\mathcal{E}\rceil}^{\mathcal{A}}$ is one of the set in the infinite sequence $q_{0}^{\prime}, q_{1}^{\prime}, \ldots$ defined by $q_{0}^{\prime}=\{L\}$ and for all $i \geq 1, q_{i}=\llbracket \varphi \rrbracket_{\lceil\mathcal{E}\rceil\left[x \mapsto q_{i-1}^{\prime}\right]}^{\mathcal{A}}$. Observe that $q_{0}^{\prime}=\left\lceil q_{0}\right\rceil$. By induction, assume that $q_{i-1}^{\prime}=\left\lceil q_{i-1}\right\rceil$ for some $i \geq 1$. Then clearly $q_{i}^{\prime}=\left\lceil q_{i}\right\rceil$ as $\lceil\mathcal{E}\rceil\left[x \mapsto q_{i-1}^{\prime}\right]=\left\lceil\mathcal{E}\left[x \mapsto q_{i-1}\right]\right\rceil$.

- if $\varphi \equiv \mu x \cdot \varphi_{1}$ and $\left\lceil\llbracket \varphi_{1} \rrbracket_{\mathcal{E}^{\prime}}^{\mathcal{S}}\right\rceil=\llbracket \varphi_{1} \rrbracket_{\left.\mathcal{F}^{\prime}\right\rceil}^{\mathcal{A}}$ for any downward closed valuation $\mathcal{E}^{\prime}$. The proof is similar to the previous case.

From Theorems 2 and 3 and Lemma 7, we can decide the existence of a deterministic observation-based sure-winning strategy for Player 1 without constructing $G^{\mathrm{K}}$.

Theorem 4 Let $G$ be a game structure of incomplete information with initial state $l_{0}$. For all $\omega$-regular objectives $\phi$, Player 1 has a deterministic observation-based strategy in $G$ for $\phi$ if and only if $\left\{l_{0}\right\} \sqsubseteq \llbracket \varphi_{\phi} \rrbracket^{\mathcal{A}}$.

Corollary 1 Let $G$ be a game structure of incomplete information. Whether a state $\ell$ of $G$ is a sure-winning state for a parity objective Parity $(p)$ can be decided in ExpTIME.

The argument to prove Corollary 1 is that the size and the number of fixed-point quantifier alternations of a $\mu$-calculus formula $\varphi$ for a parity objective $\phi$ are polynomial in the number of priorities, and the size of the parity objective $\phi$. The evaluation of $\varphi$ is exponential in the size of $\phi$. 


\section{Almost Winning}

Given a game structure $G$ of incomplete information we construct game structure $H$ by subset construction and establish certain equivalences of randomized strategies in $G$ and $H$. We then show how the reduction can be used to obtain a symbolic EXPTIME algorithm to compute almost-winning states in $G$ for Büchi objectives.

\subsection{Subset construction for almost winning}

Reduction. Given a game structure of incomplete information $G=$ $\left(L, l_{0}, \Sigma, \Delta\right.$, Obs, $\left.\gamma\right)$, we construct a game structure of complete information $H=\left(Q, q_{l_{0}}, \Sigma, \Delta_{H}\right)$ as follows: $Q=\{(s, \ell) \mid \exists o \in$ Obs. $s \subseteq \gamma(o), \ell \in s\}$; when $H$ is in a state $(s, \ell)$, it corresponds in $G$ to the state $\ell$ and the knowledge of Player 1 is $s$; the initial state is $q_{l_{0}}=\left(\left\{l_{0}\right\}, l_{0}\right)$; the transition relation $\Delta_{H} \subseteq Q \times \Sigma \times Q$ is defined as follows: $\left((s, \ell), \sigma,\left(s^{\prime}, \ell^{\prime}\right)\right) \in \Delta_{H}$ iff $\exists o \in$ Obs : $s^{\prime}=\operatorname{Post}_{\sigma}^{G}(s) \cap \gamma(o)$ and $\left(\ell, \sigma, \ell^{\prime}\right) \in \Delta$.

Plays, prefixes and cones of prefixes A play in $H$ is an infinite alternating sequence $q_{0} \sigma_{0} q_{1} \sigma_{1} \ldots$ of states in $Q$ and input letters from $\Sigma$. We denote by Play $(H)$ the set of plays of $H$. A prefix $\rho_{H}=q_{0} \sigma_{0} q_{1} \sigma_{1} \ldots \sigma_{n-1} q_{n}$ is a prefix of a play in $H$. For a prefix $\rho_{H}=q_{0} \sigma_{0} q_{1} \sigma_{1} \ldots \sigma_{n-1} q_{n}$ we denote by Last $\left(\rho_{H}\right)=q_{n}$ the last state in $\rho_{H}$ and by $\left|\rho_{H}\right|=n+1$ the length of $\rho_{H}$. We denote by $\operatorname{Pref}(H)$ the set of prefixes in $H$. For a prefix $\rho_{H} \in \operatorname{Pref}(H)$, let Cone $\left(\rho_{H}\right)=\left\{\pi_{H} \in \operatorname{Play}(H) \mid \rho_{H}\right.$ is a prefix of $\left.\pi_{H}\right\}$. The definition of Cone $\left(\rho_{G}\right)$ for prefixes $\rho_{G} \in \operatorname{Pref}(G)$ is similar.

Equivalence of states, prefixes and plays Two states $q=(s, \ell)$ and $q^{\prime}=\left(s^{\prime}, \ell^{\prime}\right)$ of $H$ are equivalent, noted $q \approx q^{\prime}$, iff $s=s^{\prime}$. Two prefixes $\rho_{H}=q_{0} \sigma_{0} q_{1} \ldots \sigma_{n-1} q_{n}$ and $\rho_{H}^{\prime}=q_{0}^{\prime} \sigma_{0}^{\prime} q_{1}^{\prime} \ldots \sigma_{n-1}^{\prime} q_{n}^{\prime}$, are equivalent, noted $\rho_{H} \approx \rho_{H}^{\prime}$, iff for all $0 \leq i \leq n$ we have $q_{i} \approx q_{i}^{\prime}$ and for all $0 \leq i \leq n-1$ we have $\sigma_{i}=\sigma_{i}^{\prime}$. Two plays $\pi_{H}, \pi_{H}^{\prime} \in \operatorname{Play}(H)$ are equivalent, noted $\pi_{H} \approx \pi_{H}^{\prime}$, iff for all $i \geq 0$ we have $\pi_{H}(i) \approx \pi_{H}^{\prime}(i)$. For a state $q \in Q$ we denote by $[q]_{\approx}=\left\{q^{\prime} \in Q \mid q \approx q^{\prime}\right\}$ the $\approx$-equivalence class of $q$. We define a similar notation for prefixes and plays.

Positional and equivalence preserving strategies. A strategy for Player 1 is a function $\alpha_{H}: \operatorname{Pref}(H) \rightarrow \mathcal{D}(\Sigma)$. A strategy $\alpha_{H}$ for Player 1 in $H$ is positional if it is independent of the prefix of plays and depends only on the last state, i.e. for all $\rho_{H}, \rho_{H}^{\prime} \in \operatorname{Pref}(H) \operatorname{such}$ that $\operatorname{Last}\left(\rho_{H}\right)=\operatorname{Last}\left(\rho_{H}^{\prime}\right)$ 


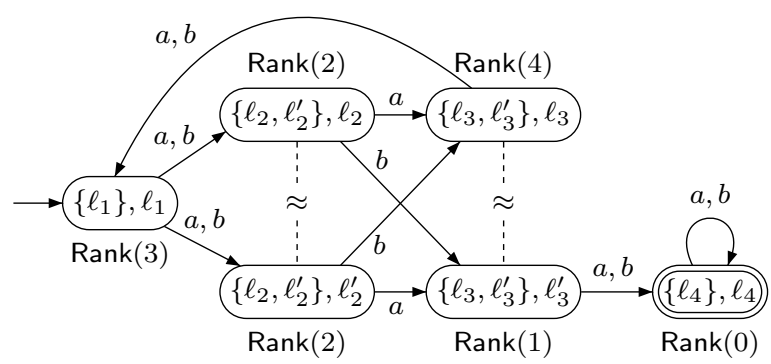

Figure 2: Game structure $H$ (for $G$ of Figure 1).

we have $\alpha_{H}\left(\rho_{H}\right)=\alpha_{H}\left(\rho_{H}^{\prime}\right)$. A positional strategy $\alpha_{H}$ can be viewed as a function $\alpha_{H}: Q \rightarrow \mathcal{D}(\Sigma)$. A strategy $\alpha_{H}$ for Player 1 is equivalence preserving if for all $\rho_{H}, \rho_{H}^{\prime} \in \operatorname{Pref}(H)$ such that $\rho_{H} \approx \rho_{H}^{\prime}$ we have $\alpha_{H}\left(\rho_{H}\right)=\alpha_{H}\left(\rho_{H}^{\prime}\right)$. We denote by $\mathcal{A}_{H}, \mathcal{A}_{H}^{P}, \mathcal{A}_{\tilde{H}}$ the set of all strategies, the set of all positional strategies, and the set of all equivalence preserving strategies for Player 1 , respectively. We denote by $\mathcal{A}_{H}^{(P)}=\mathcal{A}_{\tilde{H}} \cap \mathcal{A}_{H}^{P}$ the set of equivalence preserving positional strategies. A strategy for Player 2 is a function $\beta_{H}: \operatorname{Pref}(H) \times \Sigma \rightarrow \mathcal{D}(Q)$ such that for all $\rho_{H} \in \operatorname{Pref}(H)$, for all $\sigma \in \Sigma$, $\operatorname{Supp}\left(\beta_{H}\left(\rho_{H}, \sigma\right)\right) \subseteq \operatorname{Post}_{\text {Last }}^{H}\left(()\left(\rho_{H}\right),\{\sigma\}\right)$. We denote by $\mathcal{B}_{H}$ the set of all Player 2 strategies.

Objectives. Objectives $\Phi_{H}$ in $H$ are subsets of $(Q \times \Sigma)^{\omega}$. The definition of objectives are similar to as in Section 2. An objective $\Phi_{H}$ is equivalence preserving if for all $\pi_{H} \in \Phi_{H},\left[\pi_{H}\right] \approx \subseteq \Phi_{H}$.

Example 2 Given the game structure $G$ of incomplete information $G$ of Figure 1 the game structure $H$ produced by the reduction of Section 4 is shown in Figure 2. All the states are almost-winning for the Büchi objective $\operatorname{Buchi}\left(\left\{\left(\left\{\ell_{4}\right\}, \ell_{4}\right)\right\}\right)$. For a state $q$ we say $\operatorname{Rank}(q)=j$ if $q \in \operatorname{Rank}(j) \backslash$ $\operatorname{Rank}(j-1)$ (see notations after Lemma 11 for definition of Rank). The rank of the states as obtained from Lemma 12 are shown along side the states. The positional strategy that plays both $a$ and $b$ with equal probability is almostwinning for all states. For states $q$ of $\operatorname{Rank}(1), \operatorname{Rank}(3)$ and $\operatorname{Rank}(4)$ we have if $\operatorname{Rank}(q)=j$, then $\operatorname{Post}_{a}^{H}(q) \subseteq \operatorname{Rank}(j-1)$ and $\operatorname{Post}_{b}^{H}(q) \subseteq \operatorname{Rank}(j-1)$. For states with $\operatorname{Rank}(2)$ we have if $q=\left(\left\{\ell_{2}, \ell_{2}^{\prime}\right\}, \ell_{2}\right)$, then $\operatorname{Post}_{b}^{H}(q) \subseteq \operatorname{Rank}(1)$; and if $q=\left(\left\{\ell_{2}, \ell_{2}^{\prime}\right\}, \ell_{2}^{\prime}\right)$, then $\operatorname{Post}_{a}^{H}(q) \subseteq \operatorname{Rank}(1)$.

Mapping of prefixes and plays. We define a mapping $h: \operatorname{Pref}(G) \rightarrow$ $\operatorname{Pref}(H)$ that maps prefixes in $G$ to prefixes in $H$ as follows: given $\rho_{G}=$ 
$\ell_{0} \sigma_{0} \ell_{1} \sigma_{1} \ldots \sigma_{n-1} \ell_{n}$ we have $h\left(\rho_{G}\right)=q_{0} \sigma_{0} q_{1} \sigma_{1} \ldots \sigma_{n-1} q_{n}$, where $q_{i}=\left(s_{i}, \ell_{i}\right)$ and $s_{i}=\mathrm{K}(\mathrm{Obs}(\rho(i)))$. The mapping $h$ for plays is defined similarly.

Proposition 2 The mapping $h$ is a bijection.

Mapping of strategies for Player 1. Given a strategy $\alpha_{H}$ in $H$ we construct a strategy $\alpha_{G}=h\left(\alpha_{H}\right)$ as follows: given $\rho_{G} \in \operatorname{Pref}(G)$ we have $\alpha_{G}\left(\rho_{G}\right)=\alpha_{H}\left(h\left(\rho_{G}\right)\right)$. Similarly given a strategy $\alpha_{G}$ in $G$ we construct a strategy $\alpha_{H}=g\left(\alpha_{G}\right)$ as follows: given $\rho_{H} \in \operatorname{Pref}(H)$ we have $\alpha_{H}\left(\rho_{H}\right)=$ $\alpha_{G}\left(h^{-1}\left(\rho_{H}\right)\right)$.

Proposition 3 The following properties hold.

1. For the strategy mapping $h: \mathcal{A}_{H} \rightarrow \mathcal{A}_{G}$ we have

(a) if $\operatorname{Obs}\left(\rho_{G}\right)=\operatorname{Obs}\left(\rho_{G}^{\prime}\right)$, then $h\left(\rho_{G}\right) \approx h\left(\rho_{G}^{\prime}\right)$; and

(b) if $\alpha_{H}$ is equivalence preserving, then $h\left(\alpha_{H}\right)$ is observation-based.

2. For the strategy mapping $g: \mathcal{A}_{G} \rightarrow \mathcal{A}_{H}$ we have

(a) if $\rho_{H} \approx \rho_{H}^{\prime}$, then $\operatorname{Obs}\left(h^{-1}\left(\rho_{H}\right)\right)=\operatorname{Obs}\left(h^{-1}\left(\rho_{H}^{\prime}\right)\right)$; and

(b) if $\alpha_{G}$ is observation-based, then $g\left(\alpha_{G}\right)$ is equivalence preserving.

Mapping of strategies for Player 2. Observe that for all $q \in Q, \sigma \in \Sigma$ and $\ell \in L$ we have $\left|\left\{q^{\prime}=\left(s^{\prime}, \ell\right) \mid\left(q, \sigma, q^{\prime}\right) \in \Delta_{H}\right\}\right| \leq 1$. Given a strategy $\beta_{H}$ in $H$ we construct a strategy $\beta_{G}=h\left(\beta_{H}\right)$ as follows: for $\rho_{G} \in \operatorname{Pref}(G)$, $\sigma \in \Sigma$ and $\ell \in L$ we have $\beta_{G}\left(\rho_{G}, \sigma\right)(\ell)=\beta_{H}\left(h\left(\rho_{G}\right), \sigma\right)(s, \ell)$, where $(s, \ell) \in$ $\operatorname{Post}_{\sigma}^{H}\left(\operatorname{Last}\left(h\left(\rho_{G}\right)\right)\right)$. Similarly given a strategy $\beta_{G}$ in $G$ we construct a strategy $\beta_{H}=g\left(\beta_{G}\right)$ as follows: for $\rho_{H} \in \operatorname{Pref}(H), \sigma \in \Sigma, q=(s, \ell) \in Q$ we have $\beta_{H}\left(\rho_{H}, \sigma\right)(q)=\beta_{G}\left(h^{-1}\left(\rho_{H}\right), \sigma\right)(\ell)$.

Lemma 8 The following assertions hold.

1. For all $\rho_{H} \in \operatorname{Pref}(H)$, for every equivalence preserving strategy $\alpha_{H}$, for every strategy $\beta_{H}$ we have

$$
\operatorname{Pr}_{q_{l_{0}}}^{\alpha_{H}, \beta_{H}}\left(\operatorname{Cone}\left(\rho_{H}\right)\right)=\operatorname{Pr}_{l_{0}}^{h\left(\alpha_{H}\right), h\left(\beta_{H}\right)}\left(h^{-1}\left(\operatorname{Cone}\left(\rho_{H}\right)\right)\right) .
$$

2. For all $\rho_{G} \in \operatorname{Pref}(G)$, for every observational strategy $\alpha_{G}$, for every strategy $\beta_{G}$ we have

$$
\operatorname{Pr}_{l_{0}}^{\alpha_{G}, \beta_{G}}\left(\text { Cone }\left(\rho_{G}\right)\right)=\operatorname{Pr}_{q_{l_{0}}}^{g\left(\alpha_{G}\right), g\left(\beta_{G}\right)}\left(h\left(\text { Cone }\left(\rho_{G}\right)\right)\right) .
$$


Proof. The following properties follow from the construction of strategies in Subsection 4.1.

1. For all $\rho_{H} \in \operatorname{Pref}(H)$, for every equivalence preserving strategy $\alpha_{H}$, for every strategy $\beta_{H}$ and for all $\rho_{H}^{\prime}$ such that $\left|\rho_{H}^{\prime}\right|=\left|\rho_{H}\right|+1$, we have $\operatorname{Pr}_{q_{l_{0}}}^{\alpha_{H}, \beta_{H}}\left(\operatorname{Cone}\left(\rho_{H}^{\prime}\right) \mid \operatorname{Cone}\left(\rho_{H}\right)\right)=\operatorname{Pr}_{l_{0}}^{h\left(\alpha_{H}\right), h\left(\beta_{H}\right)}\left(h^{-1}\left(\operatorname{Cone}\left(\rho_{H}^{\prime}\right)\right) \mid\right.$ $h^{-1}\left(\right.$ Cone $\left.\left.\left(\rho_{H}\right)\right)\right)$.

2. For all $\rho_{G} \in \operatorname{Pref}(G)$, for every observation-based strategy $\alpha_{G}$, for every strategy $\beta_{G}$ and for all $\rho_{G}^{\prime}$ such that $\left|\rho_{G}^{\prime}\right|=\left|\rho_{G}\right|+1$, we have $\operatorname{Pr}_{l_{0}}^{\alpha_{G}, \beta_{G}}\left(\operatorname{Cone}\left(\rho_{G}^{\prime}\right) \mid \operatorname{Cone}\left(\rho_{G}\right)\right)=\operatorname{Pr}_{q_{l_{0}}}^{g\left(\alpha_{G}\right), g\left(\beta_{G}\right)}\left(h\left(\operatorname{Cone}\left(\rho_{G}^{\prime}\right)\right) \mid\right.$ $h\left(\right.$ Cone $\left.\left.\left(\rho_{G}\right)\right)\right)$.

The proof for the first part is as follows: observe that

$$
\begin{aligned}
\operatorname{Pr}_{q_{l_{0}}}^{\alpha_{H}, \beta_{H}}\left(\text { Cone }\left(q_{l_{0}}\right)\right) & \left.=\operatorname{Pr}_{l_{0}}^{g\left(\alpha_{H}\right), g\left(\beta_{H}\right)}\left(h^{-1} \text { Cone }\left(q_{0}\right)\right)\right) \\
& =\operatorname{Pr}_{l_{0}}^{g\left(\alpha_{H}\right), g\left(\beta_{H}\right)}\left(\text { Cone }\left(l_{0}\right)\right)=1 .
\end{aligned}
$$

The rest follows from the above properties and induction. The proof for the second part is similar.

Theorem 5 For all incomplete information game structures $G$, all Borel objectives $\Phi_{G}$ and for all equivalence preserving Borel objectives $\Phi_{H}$, for all observation-based player 1 strategies $\alpha_{G}$, for all equivalence preserving player 1 strategies $\alpha_{H}$, for all player 2 strategies $\beta_{G}$ and $\beta_{H}$ we have

$$
\begin{gathered}
\operatorname{Pr}_{l_{0}}^{\alpha_{G}, \beta_{G}}\left(\Phi_{G}\right)=\operatorname{Pr}_{q_{0}}^{g\left(\alpha_{G}\right), g\left(\beta_{G}\right)}\left(h\left(\Phi_{G}\right)\right) ; \\
\operatorname{Pr}_{q_{0}}^{\alpha_{H}, \beta_{H}}\left(\Phi_{H}\right)=\operatorname{Pr}_{l_{0}}^{h\left(\alpha_{G}\right), h\left(\beta_{G}\right)}\left(h^{-1}\left(\Phi_{G}\right)\right) .
\end{gathered}
$$

Proof. The proof is as follows: by the Caratheódary unique extension theorem a probability measure defined on Cone's has an unique extension to all Borel objectives. The result then follows from Lemma 8 and the unique extension theorem.

Corollary 2 follows from Theorem 5 .

Corollary 2 For all Borel objectives $\Phi_{G}$ we have

$$
\begin{aligned}
& \sup _{\alpha_{G} \in \mathcal{A}_{G}^{O}} \inf _{\beta_{G} \in \mathcal{B}_{G}} \operatorname{Pr}_{l_{0}}^{\alpha_{G}, \beta_{G}}\left(\Phi_{G}\right)=\sup _{\alpha_{H} \in \mathcal{A}_{\tilde{H}}} \inf _{\beta_{H} \in \mathcal{B}_{H}} \operatorname{Pr}_{q_{l_{0}}}^{\alpha_{H}, \beta_{H}}\left(h\left(\Phi_{G}\right)\right) ; \\
& \exists \alpha_{G} \in \mathcal{A}_{G}^{O} . \forall \beta_{G} \in \mathcal{B}_{G} . \quad \operatorname{Pr}_{l_{0}}^{\alpha_{G}, \beta_{G}}\left(\Phi_{G}\right)=1 \\
& \text { iff } \exists \alpha_{H} \in \mathcal{A} \widetilde{H^{*}} . \forall \beta_{H} \in \mathcal{B}_{H} \cdot \operatorname{Pr}_{q_{l_{0}}}^{\alpha_{H}, \beta_{H}}\left(h\left(\Phi_{G}\right)\right)=1 \text {. }
\end{aligned}
$$




\subsection{Almost winning for Büchi objectives}

We first illustrate the need of memory and randomization for almost-winning in incomplete information games with Büchi objectives.

Example 3 (Memory is needed to almost-win) Consider the example of Figure 3. The objective of Player 1 is to reach a state with observation $o_{4}$. We show that Player 1 has no observation based sure-winning strategy

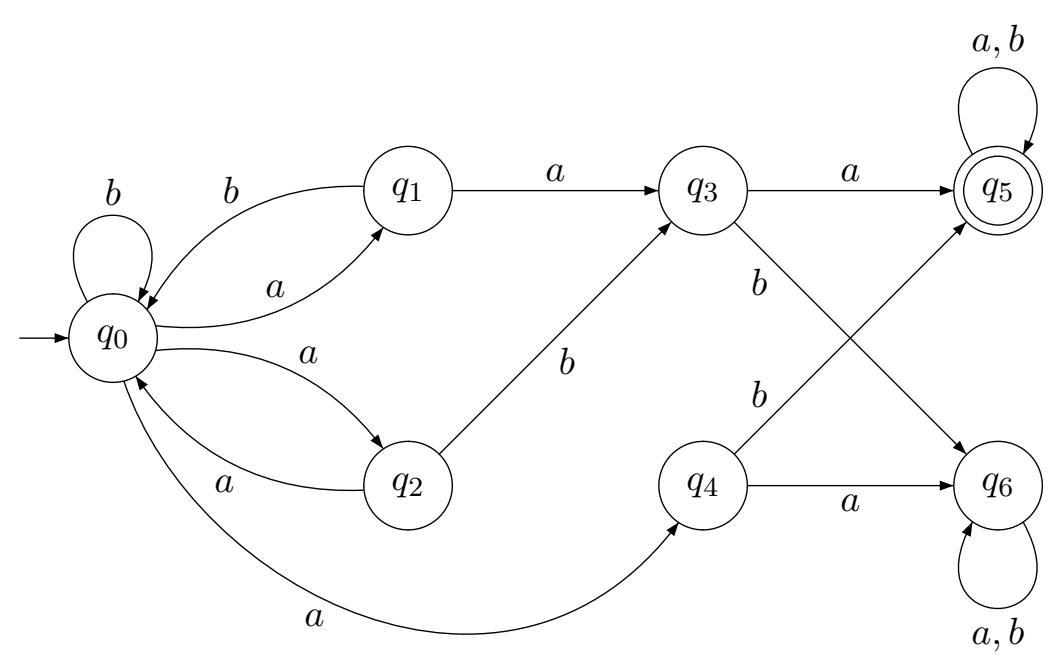

Figure 3: The observations are as follows: $o_{1}=\left\{q_{0}\right\}, o_{2}=\left\{q_{1}, q_{2}\right\}, o_{3}=$ $\left\{q_{3}, q_{4}\right\}, o_{4}=\left\{q_{5}\right\}, o_{5}=\left\{q_{6}\right\}$.

in this game. This is because when we fix an observation-based strategy for Player 1, Player 2 has a spoiling strategy to maintain the game into the states $\left\{q_{0}, q_{1}, q_{2}\right\}$. Indeed, at $q_{0}$, the only reasonable choice for Player 1 is to play a. Then Player 2 can choose to go either in $q_{1}$ or $q_{2}$. In both cases, the observation will be the same for Player 1. After seeing $o_{1} a_{2}$, if the strategy of Player 1 is to play a then Player 2 chooses $q_{2}$, otherwise, if Player 1 strategy is to play $b$ then Player 2 chooses $q_{1}$. This can be repeated and so Player 2 has a spoiling strategy against any observation based strategy of Player 1.

We now show that almost-winning strategies exist for Player 1. Consider that Player 1 plays an observation-based randomized strategy $\alpha$ as follows: after a sequence of observations $\tau$, 
- in any observation sequence $\tau$ such that $\operatorname{Last}(\tau)=o_{1}, \alpha(\tau)(a)=1$ and $\alpha(\tau)(b)=0$,

- in any observation sequence $\tau$ such that Last $(\tau)=o_{2}, \alpha(\tau)(a)=0.5$ and $\alpha(\tau)(b)=0.5$,

- in any observation sequence $\tau=\tau^{\prime} \cdot o_{1} \cdot \sigma \cdot o_{3}, \alpha(\tau)(a)=1$ and $\alpha(\tau)(b)=$ 0 ,

- in any observation sequence $\tau=\tau^{\prime} \cdot o_{2} \cdot \sigma \cdot o_{3}, \alpha(\tau)(a)=0$ and $\alpha(\tau)(b)=$ 1 ,

- otherwise take, arbitrarily, $\alpha(\tau)(a)=1$ and $\alpha(\tau)(b)=0$.

The strategy $\alpha$ is almost-winning against any randomized strategy of Player 2. Note that the strategy $\alpha$ uses memory and this is necessary because when receiving observation $o_{3}$, Player 1 has to play a if the game was just before in a state satisfying observation $o_{1}$ and $b$ if the game was just before in state satisfying $\mathrm{O}_{2}$.

Given a game structure $G$ let $H$ be the corresponding game structure constructed by the reduction. Given $O \subseteq$ Obs let $B_{O}=\{q=(s, l) \in Q \mid$ $\exists o \in O . s \subseteq \gamma(o)\}$. We have $h(\operatorname{Buchi}(O))=\operatorname{Buchi}\left(B_{O}\right)=\left\{\pi_{H} \in \operatorname{Play}(H) \mid\right.$ $\left.\operatorname{lnf}\left(\pi_{H}\right) \cap B_{O} \neq \emptyset\right\}$. We first show that almost-winning for Büchi objectives wrt to equivalence preserving strategies is equivalent to almost-winning wrt to equivalence preserving positional strategies. Formally, for $B_{O} \subseteq Q$, let

$$
\begin{aligned}
& Q \widetilde{\mathrm{AS}}=\left\{q \in Q \mid \exists \alpha_{H} \in \mathcal{A}_{\tilde{H}} \cdot \forall \beta_{H} \in \mathcal{B}_{H} \cdot \forall q^{\prime} \in[q]_{\approx} \cdot \operatorname{Pr}_{q^{\prime}}^{\beta_{H}, \beta_{H}}\left(\operatorname{Buchi}\left(B_{O}\right)\right)=1\right\} ; \\
& Q_{\mathrm{AS}}^{\approx(P)}=\left\{q \in Q \mid \exists \alpha_{H} \in \mathcal{A}_{H}^{\approx(P)} \cdot \forall \beta_{H} \in \mathcal{B}_{H} \cdot \forall q^{\prime} \in[q]_{\approx} \cdot \operatorname{Pr}_{q^{\prime}}^{\beta_{H}, \beta_{H}}\left(\operatorname{Buchi}\left(B_{O}\right)\right)=1\right\} .
\end{aligned}
$$

We will prove that $Q \widetilde{\widetilde{\mathrm{AS}}}=Q_{\mathrm{AS}}^{\approx(P)}$. Proposition 4 follows from the construction of $H$ from $G$; and yields Lemma 9 .

Proposition 4 For all $q \in Q$, for all $q_{1} \in[q]_{\approx}$, for all $\sigma \in \Sigma$, if $\left(q_{1}, \sigma, q_{1}^{\prime}\right) \in$ $\Delta_{H}$, then for all $q_{2}^{\prime} \in\left[q_{1}^{\prime}\right]_{\approx}$, there exists $q_{2} \in\left[q_{1}\right]_{\approx}$ such that $\left(q_{2}, \sigma, q_{2}^{\prime}\right) \in \Delta_{H}$.

Lemma 9 Given an equivalence preserving strategy $\alpha_{H}$, a prefix $\rho_{H} \in$ $\operatorname{Pref}(H)$ and a state $q$, if there exists a strategy $\beta_{H}$ such that $\operatorname{Pr}_{q}^{\alpha_{H}, \beta_{H}}\left(\operatorname{Cone}\left(\rho_{H}\right)\right)>0$, then for every $\rho_{H}^{\prime}$ such that $\rho_{H}^{\prime} \approx \rho_{H}$ there exists a strategy $\beta_{H}^{\prime}$ and a state $q^{\prime} \in[q] \approx$ such that $\operatorname{Pr}_{q^{\prime}}^{\alpha_{H}, \beta_{H}^{\prime}}\left(\operatorname{Cone}\left(\rho_{H}^{\prime}\right)\right)>0$. 
Observe that

$Q \backslash Q \widetilde{\widetilde{A S}}=\left\{q \in Q \mid \forall \alpha_{H} \in \mathcal{A} \widetilde{\widetilde{H}} \cdot \exists \beta_{H} \in \mathcal{B}_{H} \cdot \exists q^{\prime} \in[q]_{\approx} \cdot \operatorname{Pr}_{q^{\prime}}^{\alpha_{H}, \beta_{H}}\left(\operatorname{Buchi}\left(B_{O}\right)\right)<1\right\}$.

It follows from Lemma 9 that if a play starts in $Q \widetilde{\mathrm{AS}}$ and reaches $\left(Q \backslash Q \widetilde{\tilde{A}_{S}}\right)$ with positive probability, then for all equivalence preserving strategies for Player 1, there is a Player 2 strategy that ensures that the Büchi objective $\operatorname{Buchi}\left(B_{O}\right)$ is not satisfied with probability 1 .

Notations. For a state $q \in Q$ and $Y \subseteq Q$ we denote by $\operatorname{Allow}(q, Y)=$ $\left\{\sigma \in \Sigma \mid \operatorname{Post}_{\sigma}^{H}(q) \subseteq Y\right\}$. For a state $q \in Q$ and $Y \subseteq Q$ we denote by $\operatorname{Allow}([q], Y)=\bigcap_{q^{\prime} \in[q] \approx} \operatorname{Allow}\left(q^{\prime}, Y\right)$.

Lemma 10 For all $q \in Q \widetilde{\widetilde{\mathrm{AS}}}$ we have Allow $\left([q], Q_{\mathrm{AS}}\right) \neq \emptyset$.

Proof. Assume towards contradiction that there exists $q \in Q$ A such that Allow $([q], Q \widetilde{\mathrm{AS}})=\emptyset$. Then for all $\sigma \in \Sigma$ there exists $q^{\prime} \in[q]_{\approx}$ such that $\operatorname{Post}_{\sigma}^{H}\left(q^{\prime}\right) \cap(Q \backslash Q \widetilde{\text { AS }}) \neq \emptyset$. Hence for all equivalence preserving strategy $\alpha_{H}$ there exists $q^{\prime} \in[q]_{\approx}$ such that $\alpha_{H}\left(q^{\prime}\right)(\sigma)>0$ and $\operatorname{Post}_{\sigma}^{H}\left(q^{\prime}\right) \cap(Q \backslash$ $\left.Q \widetilde{\tilde{A S}_{\mathrm{S}}}\right) \neq \emptyset$. Hence for every equivalence strategy $\alpha_{H}$ there is a state $q^{\prime} \in[q] \approx$ and a strategy $\beta_{H}$ for Player 2 such that $Q \backslash Q \widetilde{\mathrm{AS}}$ is reached with positive probability. This contradicts that $[q]_{\approx} \subseteq Q_{\mathrm{A} S}$.

Lemma 11 Given $q \in Q \widetilde{\widetilde{A S}}$ let $\alpha_{H}$ be an equivalence preserving strategy such that for all $\beta_{H}$ and for all $q^{\prime} \in[q]_{\approx}$ we have $\operatorname{Pr}_{q^{\prime}}^{\alpha_{H}, \beta_{H}}\left(\operatorname{Buchi}\left(B_{O}\right)\right)=1$. For all $\rho_{H}=q_{0} \sigma_{0} q_{1} \ldots \sigma_{n-1} q_{n} \in \operatorname{Pref}(H)$ such that for all $0 \leq i \leq n$, $q_{i} \in Q \widetilde{\mathrm{AS}}$, if there is a strategy $\beta_{H}$ such that $\operatorname{Pr}_{q^{\prime}}^{\alpha_{H}, \beta_{H}}\left(\operatorname{Cone}\left(\rho_{H}\right)\right)>0$, then $\operatorname{Supp}\left(\alpha_{H}\left(\rho_{H}\right)\right) \subseteq \operatorname{Allow}([q], Q \widetilde{\mathrm{AS}})$.

Proof. Assume towards contradiction for a history $\rho_{H}$ satisfying the conditions of the lemma there exists $\sigma \in \operatorname{Supp}\left(\alpha_{H}\left(\rho_{H}\right)\right) \backslash$ Allow $([q], Q \widetilde{\text { AS }})$. Then there exists $q_{n}^{\prime} \in[q]_{Q \widetilde{\widetilde{A}}}$ such that $\operatorname{Post}_{\sigma}^{H}\left(q_{n}^{\prime}\right) \cap(Q \backslash Q \widetilde{\mathrm{AS}}) \neq \emptyset$. Then there exists $\rho_{H}^{\prime}$ such that $\rho_{H} \approx \rho_{H}^{\prime}$ and $\operatorname{Last}\left(\rho_{H}^{\prime}\right)=q_{n}^{\prime}$. Then by Lemma 9 there exists a strategy $\beta_{H}^{\prime}$ and $q^{\prime} \in[q]_{\approx}$ such that $\operatorname{Pr}_{q^{\prime}}^{\alpha_{H}, \beta_{H}^{\prime}}\left(\operatorname{Cone}\left(\rho_{H}^{\prime}\right)\right)>0$. Then given $\rho_{H}^{\prime}$ and the strategy $\alpha_{H}$ there exists a Player 2 strategy such that $Q \backslash Q \widetilde{\widetilde{A S}}$ is reached with positive probability. This contradicts that $\alpha_{H}$ is an almost-winning strategy.

Notations. We inductively define ranks of the states in $Q_{\mathrm{AS}}$ as follows: let $\operatorname{Rank}(0)=B_{O} \cap Q \widetilde{\mathrm{AS}}$, and

$$
\operatorname{Rank}(j+1)=\operatorname{Rank}(j) \cup\left\{\quad q \in Q \widetilde{\widetilde{A S}} \mid \exists \sigma \in \operatorname{Allow}\left([q], Q_{\widetilde{A S}}\right) \cdot \operatorname{Post}_{\sigma}^{H}(q) \subseteq \operatorname{Rank}(j)\right\} .
$$


Let $j^{*}=\min \{i \mid \operatorname{Rank}(i)=\operatorname{Rank}(i+1)\}$, and $Q^{*}=\operatorname{Rank}\left(j^{*}\right)$.

Lemma $12 Q^{*}=Q \widetilde{\widetilde{A} S}$.

Proof. Clearly by definition $Q^{*} \subseteq Q_{\overline{\mathrm{AS}}}$. We now prove that $Q_{\mathrm{A} S} \subseteq Q^{*}$. Assume towards contradiction $X=Q \widetilde{\widetilde{A} S} \backslash Q^{*} \neq \emptyset$. For all states $q \in X$, for all $\sigma \in \operatorname{Allow}([q], Q \widetilde{\text { AS }})$, we have $\operatorname{Post}_{\sigma}^{H}(q) \cap X \neq \emptyset$; as otherwise $q$ would have been in $Q^{*}$. Hence for every state $q \in X$ and $\sigma \in \operatorname{Allow}([q], Q \widetilde{\text { AS }})$ there exists $q^{\prime} \in X$ and $\left(q, \sigma, q^{\prime}\right) \in \Delta_{H}$. Fix a strategy $\beta_{H}$ for Player 2 as follows: for a state $q \in X$ and the input letter $\sigma \in \operatorname{Allow}([q], Q \widetilde{\mathrm{AS}})$ choose a successor $q^{\prime} \in X$ such that $\left(q, \sigma, q^{\prime}\right) \in \Delta_{H}$. Consider a state $q \in X$ and an equivalence preserving almost-winning strategy for Player 1 from $q$. By Lemma 11, the strategy $\alpha_{H}$ satisfies that for histories $\rho_{H}=q_{0} \sigma_{0} q_{1} \ldots \sigma_{n-1} q_{n}$ (satisfying the conditions of Lemma 11) we have $\operatorname{Supp}\left(\alpha_{H}\left(\rho_{H}\right)\right) \subseteq \operatorname{Allow}\left(\left[q_{n}\right], Q \widetilde{\mathrm{AS}}\right)$. Hence it follows that $\operatorname{Pr}_{q}^{\alpha_{H}, \beta_{H}}(\operatorname{Safe}(X))=1$. Since $B_{O} \cap Q \widetilde{\mathrm{AS}} \subseteq Q^{*}$, it follows that $B_{O} \cap X=\emptyset$. Hence $\operatorname{Pr}_{q}^{\alpha_{H}, \beta_{H}}\left(\operatorname{Reach}\left(B_{O}\right)\right)=0$, and $\operatorname{Pr}_{q}^{\alpha_{H}, \beta_{H}}\left(\operatorname{Buchi}\left(B_{O}\right)\right)=$ 0 . This contradicts that $\alpha_{H}$ is an equivalence preserving almost-winning strategy.

An equivalence-preserving positional strategy. Consider the equivalence preserving positional strategy $\alpha_{H}^{p}$ defined as follows: for a state $q \in Q \widetilde{\widetilde{\mathrm{AS}}}$ play all the moves in $\operatorname{Allow}([q], Q \widetilde{\mathrm{AS}})$ uniformly at random.

Lemma 13 For all states $q \in Q \widetilde{\mathrm{AS}}$, for all strategies $\beta_{H}$ we have:

- $\operatorname{Pr}_{q}^{\alpha_{H}^{p}, \beta_{H}}(\operatorname{Safe}(Q \widetilde{\mathrm{AS}}))=1$; and

- $\operatorname{Pr}_{q}^{\alpha_{H}^{p}, \beta_{H}}\left(\operatorname{Reach}\left(B_{O} \cap Q \widetilde{\widetilde{A S}}\right)\right)=1$.

Proof. By Lemma 12 we have $Q^{*}=Q \widetilde{\mathrm{A} S}$. Let $z=\left|Q^{*}\right|$.

- For a state $q \in Q \widetilde{\mathrm{AS}}$ we have $\operatorname{Post}_{\sigma}^{H}(q) \subseteq Q \widetilde{\mathrm{ASS}}$ for all $\sigma \in \operatorname{Allow}\left([q], Q_{\widetilde{\mathrm{AS}}}\right)$. Hence it follows for all states $q \in Q \widetilde{\mathrm{AS}}$, for all strategies $\beta_{H}$ for Player 2 we have $\operatorname{Pr}_{q}^{\alpha_{H}^{p}, \beta_{H}}(\operatorname{Safe}(Q \widetilde{\mathrm{AS}}))=1$.

- For a state $q \in(\operatorname{Rank}(j+1) \backslash \operatorname{Rank}(j))$, there exists $\sigma \in \operatorname{Allow}([q], Q \widetilde{\mathrm{AS}})$ such that $\operatorname{Post}_{\sigma}^{H}(q) \subseteq \operatorname{Rank}(j)$. For a set $Y \subseteq Q$ let $\diamond^{j}(Y)$ denote the set of paths that reaches $Y$ within $j$ steps. It follows that for all states $q \in \operatorname{Rank}(j+1)$, for all strategies $\beta_{H}$ we have

$$
\operatorname{Pr}_{q}^{\alpha_{H}^{p}, \beta_{H}}\left(\diamond^{1}(\operatorname{Rank}(j))\right) \geq \frac{1}{|\Sigma|} .
$$


Let $B=B_{O} \cap Q \widetilde{\mathrm{A} S}$. By induction on the ranks it follows that for all states $q \in Q^{*}$, for all strategies $\beta_{H}$ we have

$$
\operatorname{Pr}_{q}^{\alpha_{H}^{p}, \beta_{H}}\left(\diamond^{z}(\operatorname{Rank}(0))\right)=\operatorname{Pr}_{q}^{\alpha_{H}^{p}, \beta_{H}}\left(\diamond^{z}(B)\right) \geq\left(\frac{1}{|\Sigma|}\right)^{z}=r>0 .
$$

For $m>0$ we have $\operatorname{Pr}_{q}^{\alpha_{H}^{p}, \beta_{H}}\left(\diamond^{m \cdot z}(B)\right) \geq 1-(1-r)^{m}$. Thus we have $\operatorname{Pr}_{q}^{\alpha_{H}^{p}, \beta_{H}}(\operatorname{Reach}(B))=\lim _{m \rightarrow \infty} \operatorname{Pr}_{q}^{\alpha_{H}^{p}, \beta_{H}}\left(\diamond^{m \cdot z}(B)\right) \geq \lim _{m \rightarrow \infty} 1-(1-r)^{m}=1$.

The result follows.

It follows from Lemma 13 that given the strategy $\alpha_{H}^{p}$, the set $Q \widetilde{\mathrm{AS}}$ is never left and the states in $B_{O} \cap Q \widetilde{\mathrm{AS}}$ are reached with probability 1 . Since this happens for every state in $Q \widetilde{\mathrm{AS}}$ it follows that the set $B_{O} \cap Q_{\mathrm{AS}}$ is visited infinitely often with probability 1 , i.e., the Büchi objective $\operatorname{Buchi}\left(B_{O}\right)$ is satisfied with probability 1 . The above analysis along with the fact that $\left[q_{l_{0}}\right]_{\approx}$ is a singleton and Corollary 2 proves that $Q \widetilde{\mathrm{AS}}=Q_{\mathrm{AS}}^{\approx(P)}$. Theorem 6 follows and the result also easily specializes for the case of reachability objectives.

Theorem 6 For all incomplete information game structures $G$, for a set $O$ of observations, there exists an observation-based almost-winning strategy in $G$ for the objective Buchi $(O)$ iff there exists an equivalence preserving positional almost-winning strategy in $H$ for the objective $\operatorname{Buchi}\left(B_{O}\right)$.

Symbolic algorithm We present a symbolic quadratic time (in the size of $H$ ) algorithm to compute the set $Q \widetilde{\mathrm{AS}}$. For $Y \subseteq Q$ and $X \subseteq Y$, let

$$
\begin{gathered}
\operatorname{Apre}(Y, X)=\left\{q \in Y \mid \exists \sigma \in \operatorname{Allow}([q], Y) . \operatorname{Post}_{\sigma}^{H}(q) \subseteq X\right\} ; \\
\operatorname{Spre}(Y)=\{q \in Y \mid \operatorname{Allow}([q], Y) \neq \emptyset\}=\operatorname{Apre}(Y, Y) .
\end{gathered}
$$

Let $\phi=\nu Y . \mu X$. Apre $(Y, X) \vee\left(B_{O} \wedge \operatorname{Spre}(Y)\right)$ and $Z=\llbracket \phi \rrbracket$.

Lemma $14 Z=Q \widetilde{\mathrm{AS}}$.

Proof of Lemma 14. We prove $Z=Q_{\text {ÁS }}$ by proving inclusion in both directions. We have $Z=\llbracket \phi \rrbracket$ and $\phi=\nu Y . \mu X$. (Apre $\left.(Y, X) \vee\left(B_{O} \wedge \operatorname{Spre}(Y)\right)\right)$ 
1. We first show that $Z \subseteq Q \widetilde{\mathrm{AS}}$. Since $Z$ is a fixed-point of $\phi$ we have

$$
Z=\llbracket \mu X .\left(\operatorname{Apre}(Z, X) \vee\left(B_{O} \wedge \operatorname{Spre}(Z)\right)\right) \rrbracket .
$$

We analyze the evaluation of $Z$ as the fixed-point as follows: let $X_{0}=\emptyset$ and $X_{i+1}=\operatorname{Apre}\left(Z, X_{i}\right) \vee\left(B_{O} \wedge \operatorname{Spre}(Z)\right)$. Observe that since $X_{0}=\emptyset$ we have Apre $\left(Z, X_{0}\right)=\emptyset$ and hence $X_{1}=B_{O} \cap \operatorname{Spre}(Z) \subseteq B_{O}$. Let $j^{*}=$ $\min \left\{i \mid X_{i+1}=X_{i}\right\}$ and we have $Z=X_{j^{*}}$. Consider the equivalence preserving strategy $\alpha_{H}^{p}$ for Player 1 that at a state $q \in Z$ plays all moves in $\operatorname{Allow}([q], Z)$ uniformly at random. For all strategies $\beta_{H}$ for Player 2 and for all states $q \in Z$ we have $\operatorname{Pr}_{q}^{\alpha_{H}^{p}, \beta_{H}}(\operatorname{Safe}(Z))=1$. Also for a state $q \in\left(X_{i+1} \backslash X_{i}\right) \backslash B_{O}$ we have there exists $\sigma \in$ Allow $([q], Z)$ such that $\operatorname{Post}_{\sigma}^{H}(q) \subseteq X_{i}$, i.e., for a state $q \in\left(X_{i+1} \backslash X_{i}\right) \backslash B_{O}$, given $\alpha_{H}^{p}$ against all strategies $\beta_{H}$ the next state is in $X_{i}$ with probability at least $\frac{1}{|\Sigma|}$. Arguments similar to Lemma 13 establishes that $\alpha_{H}^{p}$ is an almost-winning strategy for all states $q \in Z$. Hence we have $Z \subseteq Q \widetilde{\mathrm{A} S}$.

2. We now show that $Q_{\mathrm{A} S} \subseteq Z$. We first show that $Q_{\mathrm{A} S}$ satisfies that

$$
Q \widetilde{\widetilde{\mathrm{AS}}}=\llbracket \mu X .\left(\operatorname{Apre}\left(Q_{\mathrm{AS}}, X\right) \vee\left(B_{O} \wedge \operatorname{Spre}(Q \widetilde{\mathrm{AS}})\right)\right) \rrbracket .
$$

Observe that $Q_{\overline{\mathrm{AS}}}=\operatorname{Spre}\left(Q_{\mathrm{AS}}\right)$. We now analyze the evaluation of $Q \widetilde{\widetilde{A} S}$ as the fixed-point $Q^{*}$ as shown in Lemma 12. Let $X_{0}=\emptyset$, then $\operatorname{Apre}\left(Q_{\tilde{\mathrm{AS}}}, X_{0}\right)=\emptyset$. Hence $X_{1}=\operatorname{Apre}\left(Q_{\mathrm{ASS}}\right) \vee\left(B_{O} \wedge \operatorname{Spre}(Q \widetilde{\mathrm{AS}})\right)=$ $B_{O} \wedge Q \widetilde{\mathrm{AS}}=\operatorname{Rank}(0)$ (as defined before Lemma 12). By the definition of $\operatorname{Rank}(j+1)$ from $\operatorname{Rank}(j)$ and the definition of Apre $(\cdot, \cdot)$ and Spre $(\cdot)$ it follows that for all $i>0$, given $\operatorname{Rank}(i-1)=X_{i}$, we have $\operatorname{Rank}(i+$ $1)=X_{i}=\operatorname{Apre}\left(Q_{\mathrm{AS}}, X_{i}\right) \vee\left(B_{O} \wedge \operatorname{Spre}\left(Q_{\text {AS }}\right)\right)$. By induction we have $Q^{*}=\llbracket \mu X$. Apre $\left.\left(Q_{\tilde{\mathrm{A} S}}, X\right) \vee\left(B_{O} \wedge \operatorname{Spre}\left(Q_{\widetilde{\mathrm{A} S}}\right)\right)\right) \rrbracket$. Since $Q^{*}=Q_{\mathrm{A} S}($ by Lemma 12) we have

$$
Q \widetilde{\widetilde{\mathrm{AS}}}=\llbracket \mu X .\left(\operatorname{Apre}\left(Q_{\tilde{\mathrm{AS}}}, X\right) \vee\left(B_{O} \wedge \operatorname{Spre}(Q \widetilde{\widetilde{\mathrm{AS}}})\right)\right) \rrbracket .
$$

Since $Z$ is the greatest fixed-point we have $Q_{\mathrm{A} S} \subseteq Z$.

The result follows.

Theorem 7 Let $G$ be a game structure of incomplete information and a set $O$ of observations. Whether a state $\ell$ of $G$ is an almost-winning state for a Büchi objective $\operatorname{Buchi}(O)$ can be decided in ExpTime. 
Lemma 14 and the fact that $H$ is exponential in size of $G$ yield Theorem 7 . The arguments for the proof of Theorem 6 and Theorem 7 do not directly extend to coBüchi or parity objectives in general. In fact Theorem 6 does not hold for parity objectives in general for the following reason: in concurrent games with parity objectives with more than two priorities almost-winning strategies require infinite memory in general; for an example see [5]. Such concurrent games are reducible to semi-perfect information games [4] and semi-perfect information games are reducible to the incomplete information games we study. Hence reduction to a finite game structure and obtaining randomized memoryless strategy is not possible in general for parity objectives. Theorem 6 and Theorem 7 may hold for coBüchi objectives, but there does not seem to be a simple extension of arguments of Büchi objectives to the coBüchi case. The results similar to Theorem 6 and Theorem 7 for coBüchi objectives is open.

Direct symbolic algorithm As in Section 3.2, the structure $H$ has not to be constructed explicitly and we can obtain a direct fixed point algorithm on a well chosen lattice. The fixed point formula to compute the set $Q \widetilde{\mathrm{AS}}$ is evaluated on the lattice $\left\langle 2^{Q}, \subseteq, \cup, \cap, Q, \emptyset\right\rangle$. It is easy to show that the sets computed by the fixed point algorithm are downward closed for the following order on $Q$ : for $(s, \ell),\left(s^{\prime}, \ell^{\prime}\right) \in Q$ let $(s, \ell) \preceq\left(s^{\prime}, \ell^{\prime}\right)$ iff $\ell=\ell^{\prime}$ and $s \subseteq s^{\prime}$. Then, we can define an antichain over $Q$ as a set of pairwise $\preceq$-incomparable elements of $Q$, and compute the almost-sure winning states in the lattice of antichains over $Q$, without explicitly constructing $H$.

\section{Lower Bounds}

We show that deciding the existence of a deterministic (resp. randomized) observation-based sure-winning (resp. almost-winning) strategy for Player 1 in games of incomplete information is EXPTIME-hard already for reachability objectives. Note that the result for sure-winning follows from results in [19] but our new proof extends to almost-winning as well.

Sure winning. To show the lower bound result, we use a reduction of the membership problem for polynomial space Alternating Turing Machine. An alternating Turing machine (ATM) is a tuple $M=\left\langle Q, q_{0}, g, \Sigma_{i}, \Sigma_{t}, \delta, F\right\rangle$ where:

- $Q$ is a finite set of control states; 
- $q_{0} \in Q$ is the initial state;

- $g: Q \rightarrow\{\wedge, \vee\}$

- $\Sigma_{i}=\{0,1\}$ is the input alphabet;

- $\Sigma_{t}=\{0,1,2\}$ is the tape alphabet and 2 is the blank symbol;

- $\delta \subseteq Q \times \Sigma_{t} \times Q \times \Sigma_{t} \times\{-1,1\}$ is a transition relation; and

- $F \subseteq Q$ is the set of accepting states.

We say that $M$ is a polynomial space ATM if for some polynomial $p(\cdot)$, the space used by $M$ on any input word $w$ is bounded by $p(|w|)$.

Without loss of generality, we make the hypothesis that the initial control state of the machine is a $\vee$-state and that transitions link $\vee$-state to $\wedge$-state and vice versa. A word $w$ is accepted by an ATM $M$ if there exists a run tree of $M$ on $w$ whose all leaf nodes are accepting configurations. The AND-OR graph of the polynomial space $\operatorname{ATM}(M, p)$ on the input word $w \in \Sigma^{*}$ is $G(M, p)=\left\langle S_{\vee}, S_{\wedge}, s_{0}, \Rightarrow, R\right\rangle$ where

- $S_{\vee}=\left\{(q, h, t) \mid q \in Q, g(q)=\vee, 1 \leq h \leq p(|w|)\right.$ and $\left.t \in \Gamma^{p(|w|)}\right\} ;$

- $S_{\wedge}=\left\{(q, h, t) \mid q \in Q, g(q)=\wedge, 1 \leq h \leq p(|w|)\right.$ and $\left.t \in \Gamma^{p(|w|)}\right\}$;

- $s_{0}=\left(q_{0}, 1, t\right)$ where $t=w \cdot \Gamma^{p(|w|)-|w|}$;

- $\left(\left(q_{1}, h_{1}, t_{1}\right),\left(q_{2}, h_{2}, t_{2}\right)\right) \in \Rightarrow$ iff there exists $\left(q_{1}, t_{1}\left(h_{1}\right), q, \gamma, d\right) \in \delta$ such that $q_{2}=q, h_{2}=h_{1}+d, t_{2}\left(h_{1}\right)=\gamma$ and $t_{2}(i)=t_{1}(i)$ for all $i \neq h_{1}$;

- $R=\left\{(q, h, t) \in S_{\vee} \cup S_{\wedge} \mid q \in F\right\}$.

A word $w$ is accepted by $(M, p)$ iff $R$ is reachable in $G(M, p)$. The membership problem is to decide if a given word $w$ is accepted by a given polynomial space $\operatorname{ATM}(M, p)$. This problem is known to be ExpTime-HARD [3].

Idea of the reduction. Given a polynomial space ATM $M$ and a word $w$, we construct a game of size polynomial in the size of $(M, w)$ to simulate the execution of $M$ on $w$. Player 1 makes choices in $\vee$-states and Player 2 makes choices in $\wedge$-states. Furthermore, Player 1 is responsible for maintaining the symbol under the tape head. The objective is to reach an accepting configuration of the ATM.

Each turn proceeds as follows. In an $\vee$-state, by choosing a letter $(t, a)$ in the alphabet of the game, Player 1 reveals $(i)$ the transition $t$ of the ATM 
that he has chosen (this way he also reveals the symbol that is currently under the tape head) and (ii) the symbol $a$ under the next position of the tape head. If Player 1 lies about the current or the next symbol under the tape head, he should loose the game, otherwise the game proceeds. The machine is now in an $\wedge$-state and Player 1 has no choice: he announces a special symbol $\epsilon$ and Player 2, by resolving nondeterminism on $\epsilon$, chooses a transition of the Turing machine which is compatible with the current symbol under the tape head revealed by Player 1 at the previous turn. The state of the ATM is updated and the game proceeds. The transition chosen by Player 2 is visible in the next state of the game and so Player 1 can update his knowledge about the configuration of the ATM. Player 1 wins whenever an accepting configuration of the ATM is reached, that is $w$ is accepted.

The difficulty is to ensure that Player 1 looses when he announces a wrong content for the cell under the tape head. As the number of configurations of the polynomial ATM is exponential, we can not directly encode the full configuration of the ATM in the states of the game. To overcome this difficulty, we use the power of incomplete information as follows. Initially, Player 2 chooses a position $k, 1 \leq k \leq p(|w|)$, on the tape: this number as well as the symbol $\sigma \in\{0,1,2\}$ that lies in the tape cell number $k$ is maintained all along the game in the non-observable portion of the game states. The pair $(\sigma, k)$ is thus private to Player 2 and invisible to Player 1 . Thus, at any point in the game, Player 2 can check whether Player 1 is lying when announcing the content of cell number $k$, and go to a sink state if Player 1 cheats (no other states can be reached from there). Since Player 1 does not know which cell is monitored by Player 2 ( $k$ is private), to avoid loosing, he should not lie about any of the tape cells and thus he should faithfully simulate the machine. Then, he wins the game if and only if the ATM accepts the words $w$.

Almost winning. To establish lower bound for almost-winning, we can use the same reduction. Randomization can not help Player I in this game. Indeed, at any point of the game, if Player I takes a chance in either not faithfully simulating the ATM or lying about the symbol under the tape head, the sink state is reached. In those case, the probability to reach the sink state is positive and so the probability to win the game is strictly less than one. We now present the details of the reduction of the hardness proof. 
Reduction Given a polynomial space $\operatorname{ATM}(M, p)$, with $M=$ $\left\langle Q, q_{0}, g, \Sigma_{i}, \Sigma_{t}, \delta, F\right\rangle$ and a word $w$, we construct the following game structure $G_{M, p, w}=\left\langle L, l_{0}, \Sigma, \Delta\right.$, Obs, $\left.\gamma\right\rangle$, where:

- The set of positions $L=\{$ init $\} \cup\{$ sink $\} \cup L_{1} \cup L_{2}$ where: $L_{1}=(\delta \cup\{-\}) \times$ $Q \times\{1, \ldots, p(|w|)\} \times\{1, \ldots, p(|w|)\} \times \Sigma_{t}$. A state $(t, q, h, k, \sigma)$ consists of a transition $t$ of the ATM chosen by Player 2 at the previous round or - if this is the first round where Player 1 plays, the current control state $q$ of $M$, the position $h$ of the tape head, the pair $(k, \sigma)$ such that the $k$-th symbol of the tape is $\sigma$, this pair $(k, \sigma)$ will be kept invisible for Player 1. $L_{2}=Q \times\{1, \ldots, p(|w|)\} \times \Sigma_{t} \times\{1, \ldots, p(|w|)\} \times \Sigma_{t}$. A state $(q, h, \gamma, k, \sigma)$ consists of $q, h, k, \sigma$ as in $L_{1}$ and $\gamma$ is the symbol that Player 1 claims to be under the tape head. The objective for Player 1 will be to reach a state $l \in L$ associated with an accepting control state of $M$.

- $l_{0}=$ init

- $\Sigma=\{\epsilon\} \cup\left(\delta \times \Sigma_{t}\right)$.

- The transition relation $\Delta$ contains the following sets of transitions:

- $I_{1}$ that contains transitions (init, $\epsilon,\left(-, q_{0}, 1, k, \sigma\right)$ ) where $(i) 1 \leq$ $k \leq p(|w|)$ and $(i i) \sigma=w(k)$ if $1 \leq k \leq|w|$ and $\sigma=2$ otherwise. $I_{2}$ that contains transitions (init, $(t, \gamma)$, sink) where $(t, \gamma) \in \delta \times \Sigma_{t}$. So, at the initial state init Player 1 has to play $\epsilon$ in order to avoid entering sink. By resolving nondeterminism on $\epsilon$, Player 2 chooses a tape cell to monitor.

- $S$ that contains transitions (sink, $\sigma$, sink) for all $\sigma \in \Sigma$. When the sink state is entered, the game stays there forever.

- $L_{1.1}$ that contains transitions $((t, q, h, k, \sigma), \epsilon$, sink $) . L_{1.2}$ that contains transitions $\left((t, q, h, k, \sigma),\left(\left(q_{1}, \gamma_{1}, q_{2}, \gamma_{2}, d\right), \gamma_{3}\right)\right.$, sink) where $q_{1} \neq q$ or $\neg(1 \leq h+d \leq p(|w|)) . \quad L_{1.3}$ contains the transitions $\left((t, q, h, k, \sigma),\left(\left(q_{1}, \gamma_{1}, q_{2}, \gamma_{2}, d\right), \gamma_{3}\right)\right.$, sink) where $h=k \wedge \gamma_{1} \neq$ $\sigma$ or $h+d=k \wedge \gamma_{3} \neq \sigma . \quad L_{1.4}$ contains the transitions $\left((t, q, h, k, \sigma),\left(\left(q_{1}, \gamma_{1}, q_{2}, \gamma_{2}, d\right), \gamma_{3}\right),\left(q_{2}, h+d, \gamma_{3}, k, \sigma^{\prime}\right)\right)$ such that $q=q_{1}, 1 \leq h+d \leq p(|w|), h=k \rightarrow\left(\gamma_{1}=\sigma \wedge \sigma^{\prime}=\gamma_{2}\right)$, and $h \neq k \rightarrow \sigma^{\prime}=\sigma$. Those transitions are associated with states of the game where Player 1 chooses a transition of the ATM to execute (if he proposes $\epsilon$, the game evolves to the sink state, see $\left.L_{1.1}\right)$. The transition proposed by Player 1 should be valid for the 
current control state of the ATM and the head should not exit the bounded tape after execution of the transition by the ATM, otherwise the game evolves to the sink state, see L.1.2. When choosing a letter, Player 1 also reveals the current letter under the tape head (given by the transition) as well as the letter under the next position of the tape head. If one of those positions is the one that is monitored by Player 2, the game evolves to the sink state in case Player 1 lies, see $L_{1.3}, L_{1.4}$.

- $L_{2.1}$ contains the transitions $\left(\left(q, h, \gamma_{1}, k, \sigma\right), \epsilon,\left(\left(q_{1}, \gamma_{2}, q_{2}, \gamma_{3}, d\right), q_{3}, h+\right.\right.$ $\left.\left.d, k, \sigma^{\prime}\right)\right)$ such that $q=q_{1}, q_{2}=q_{3}, \gamma_{1}=\gamma_{2}, 1 \leq h+d \leq p(|w|)$, $h=k \rightarrow \sigma^{\prime}=\gamma_{3}$, and $h \neq k \rightarrow \sigma^{\prime}=\sigma . L_{2.2}$ contains the transitions $\left(\left(q, h, \gamma_{1}, k, \sigma\right), \epsilon\right.$, sink $)$ such that there does not exist a transition $\left(q, \gamma_{1}, q_{1}, \gamma_{2}, d\right) \in \delta$ with $1 \leq h+d \leq p(|w|)$. $L_{2.3}$ contains the transitions $\left(\left(q, h, \gamma_{1}, k, \sigma\right),(t, \gamma)\right.$, sink) where $(t, \gamma) \in \Sigma \backslash\{\epsilon\}$. Those transitions are associated with states of the game where Player 2 chooses the next transition of the ATM to execute. Player 1 should play $\epsilon$ otherwise the game goes to the sink state (see $L_{2.3}$, also the game goes to the sink state if there is no valid transition to execute in the ATM (see $L_{2.2}$ ). In the other cases, Player 1 proposes $\epsilon$ and Player 2 chooses a valid transition by resolving nondeterminism. The copy of the monitored cell is updated if necessary.

- Obs $=\{$ init, sink $\} \cup \mathrm{Obs}_{1} \cup \mathrm{Obs}_{2}$ where Obs $1=\{(t, q, h) \mid \exists(t, q, h, k, \sigma) \in$ $\left.L_{1}\right\}$ and $\mathrm{Obs}_{1}=\left\{(q, h, \gamma) \mid \exists(q, h, \gamma, k, \sigma) \in L_{2}\right\}$.

- $\gamma$ is defined as follows: $\gamma($ init $)=\{$ init $\}, \gamma($ sink $)=\{$ sink $\}$, for all $(t, q, h) \in \mathrm{Obs}_{1}, \gamma(t, q, h)=\left\{(t, q, h, k, \sigma) \in L_{1}\right\}$, for all $(q, h, \gamma) \in$ $\mathrm{Obs}_{2}, \gamma(q, h, \gamma)=\left\{(q, h, \gamma, k, \sigma) \in L_{2}\right\}$.

Finally, the objective $\phi$ of this game for Player 1 is to reach a state where the associated control state of the ATM is accepting, i.e. $\phi=$ $\left\{o_{1} o_{2} \ldots o_{n} \cdots \in \mathrm{Obs}^{\omega} \mid \exists i \geq 0:\left(o_{i}=(t, q, h) \in \mathrm{Obs}_{1} \vee o_{i}=(q, h, \gamma) \in\right.\right.$ $\left.\left.\mathrm{Obs}_{2}\right) \wedge q \in F\right\}$.

It follows that Player 1 has an observation-based sure-winning (or almost-winning) strategy in the game $G_{M, p, w}$ for the objective $\phi$ iff the word $w$ is accepted by the polynomial space $\operatorname{ATM}(M, p)$.

Theorem 8 Player 1 has a deterministic (resp. randomized) observationbased sure-winning (resp. almost-winning) strategy in the game $G_{M, p, w}$ for 
the objective $\phi$ iff the word $w$ is accepted by the polynomial space ATM $(M, p)$.

Theorem 9 Given a game structure of incomplete information $G$ with reachability objective $\phi$ and a state $\ell$, deciding (a) whether $\ell$ is a sure-winning state for $\phi$ in $G$ is EXPTIME-HARD; and (b) whether $\ell$ is an almost-winning state for $\phi$ in $G$ is EXPTIME-HARD.

\section{References}

[1] M. Abadi, L. Lamport, and P. Wolper. Realizable and unrealizable specifications of reactive systems. In ICALP'89, pages 1-17. Springer, 1989.

[2] R. Alur, T.A. Henzinger, and O. Kupferman. Alternating-time temporal logic. JACM, 49:672-713, 2002.

[3] A. K. Chandra, D. Kozen, and L. J. Stockmeyer. Alternation. JACM, 28(1):114-133, 1981.

[4] K. Chatterjee and T.A. Henzinger. Semiperfect-information games. In FSTTCS'05, pages 1-18. Springer, 2005.

[5] L. de Alfaro and T.A. Henzinger. Concurrent omega-regular games. In LICS 00, pages 141-154. IEEE Computer Society Press, 2000.

[6] L. de Alfaro and T.A. Henzinger. Interface automata. In FSE'01, pages 109120. ACM Press, 2001.

[7] L. de Alfaro, T.A. Henzinger, and O. Kupferman. Concurrent reachability games. In FOCS'98, pages 564-575. IEEE Computer Society Press, 1998.

[8] L. de Alfaro, T.A. Henzinger, and R. Majumdar. From verification to control: dynamic programs for omega-regular objectives. In $L I C S^{\prime} 01$, pages 279-290. IEEE Computer Society Press, 2001.

[9] M. De Wulf, L. Doyen, T.A. Henzinger and J.-F. Raskin. Antichains: a New Algorithm to Solve Universality of FA In $C A V$, Springer, To appear, 2006.

[10] M. De Wulf, L. Doyen, and J.-F. Raskin. A lattice theory for solving games of imperfect information. In $H S C C$, pages 153-168, Springer, 2006.

[11] D. Dill. Trace Theory for Automatic Hierarchical Verification of Speedindependent Circuits. The MIT Press, 1989.

[12] E.A. Emerson and C.S. Jutla. Tree automata, mu-calculus and determinacy (extended abstract). In FOCS'91, pages 368-377. IEEE Computer Society Press, 1991. 
[13] A. Kechris. Classical Descriptive Set Theory. Springer, 1995.

[14] O. Kupferman and M. Vardi. Synthesis with incomplete informatio. ICTL'97, 1997.

[15] M.L. Littman. Algorithms for sequential decision making. PhD Thesis, Brown University, 1996.

[16] D. Martin. Borel determinacy. Annals of Mathematics, 102(2):363-371, 1975.

[17] A. Pnueli and R. Rosner. On the synthesis of an asynchronous reactive module. In ICALP'89, pages 652-671, Springer, 1989.

[18] P. Ramadge and W. Wonham. Supervisory control of a class of discrete-event processes. SIAM Journal of Control and Optimization, 25(1):206-230, 1987.

[19] J. H. Reif. The complexity of two-player games of incomplete information. JCSS, 29(2):274-301, 1984.

[20] W. Thomas. Languages, automata, and logic. In Handbook of Formal Languages, volume 3, Beyond Words, chapter 7, pages 389-455. Springer, 1997.

[21] M. Vardi. Automatic verification of probabilistic concurrent finite-state systems. In FOCS'85, pages 327-338. IEEE Computer Society Press, 1985. 